\title{
Environmental Policy when Consumers Value Conformity ${ }^{1}$
}

\author{
Alistair Ulph ${ }^{2}$ \\ and \\ David Ulph ${ }^{3}$
}

July 2018

JEL Classification: D11, D69

Keywords: desire for conformity; participation-consistent consumption interval; distribution of types; existence of equilibrium consumption norms; policy implications.

\section{Please do not quote.}

\footnotetext{
${ }^{1}$ We are grateful to Ngo van Long, Partha Dasgupta, Dale Southerton, participants at the Bioecon 2017 conference at University of Tilburg, workshop participants at the University of Bath, University of Manchester and University of St Andrews, three anonymous referees and the editors of this special issue for comments on earlier versions of this paper. The usual disclaimer applies.

${ }^{2}$ Professor of Economics, School of Social Sciences and Sustainable Consumption Institute, University of Manchester. E-mail: Alistair.Ulph@manchester.ac.uk; Tel: +44 (0)161 3066000 Fax: +44 (0)1612750188

${ }^{3}$ Professor of Economics, School of Economics \& Finance, University of St Andrews. E-mail: du1@st-andrews.ac.uk; Tel: +44 (0)1334 462425 Fax:+44 (0)1334 462444
} 


\begin{abstract}
We present a model of consumer behaviour when consumers value conformity and examine the implications for environmental policy. The model shares a feature set out in Dasgupta, Southerton, Ulph and Ulph (2016) of having a structure of preferences for conformity which induces a mass of consumers to adhere exactly to a norm level of consumption (clumping). However we extend our previous analysis by analysing the conditions for the existence and potential uniqueness of consumption norms. In doing so we introduce threshold effects whereby individuals adhere to a norm only if sufficiently many others do so. Taken together these have striking implications for environmental policy in the case where the norm good generates pollution emissions. Clumping means many individuals will not change behaviour unless the norm changes while threshold effects plus clumping means that it may be hard to change a norm. We show that the use of Pigovian taxes to control behaviour may be either ineffective or welfare reducing, and that the optimal Pigovian tax will work only if it is above some threshold level. There are parameter values for which quantity-based injunctive policies raise welfare relative to no intervention while optimal Pigovian taxes would lower welfare.
\end{abstract}




\section{Introduction}

In this paper we contribute to the growing literature on the design of environmental policy when the behaviour of individuals is driven not by self-regarding preferences but by a recognition that they are part of a wider society - a recognition that can shape their behaviour in a number of different ways ${ }^{1}$. In particular we set out a model for exploring the policy implications of assuming that individual consumption is determined not just by the standard flow of utility from own consumption but also by a desire to conform with the consumption decisions of other individuals - that is to adhere to some norm level of consumption. In contrast with the standard analysis of Veblen effects which produces over-consumption, ${ }^{2}$ a desire for conformity implies that while some individuals may adhere to a consumption norm by raising their consumption, others will cut it. As in Dasgupta, Southerton, Ulph and Ulph (2016) we assume that structure of preferences is such that, if a consumption norm exists, there will be a set of individuals of positive measure that will adhere exactly to the norm level of consumption ${ }^{3}$. It is this clumping of behaviour that in our view identifies the presence of a norm as distinct from just a more concentrated distribution of preferences.

However we extend the analysis in the previous paper by examining more systematically the conditions under which a norm level of consumption might exist and whether there could be equilibria in which more than one such norm level could co-exist ${ }^{4}$. In doing so we draw on another idea in behavioural economics - that of threshold behaviour - by assuming that individuals will only adhere to a norm if enough other people do so.

These two features of our model have important consequences. The clumping of consumption means that there will be many individuals who will not change their behaviour unless there is a change in the norm level of consumption, while the threshold effect, combined with clumping, means that it may be very hard to shift the norm level of consumption.

\footnotetext{
${ }^{1}$ See for example Dasgupta, Southerton, Ulph and Ulph (2016) and the references therein. ${ }^{2}$ For recent analyses of the Veblen effect see Arrow and Dasgupta (2010), Dasgupta, Southerton, Ulph and Ulph (2016) and Ulph (2014). The Veblen effect is invoked to explain the Easterlin Paradox (Easterlin (1974, 2001) ) whereby, after a certain level of per capita income, further growth in income per capita seems to have no effect on measures of well-being as captured by surveys of happiness (see for example Blanchflower and Oswald (2004)).

${ }^{3}$ In other models of consumption norms - e.g. Bernheim (1994) - the only individuals who would adhere exactly to the norm level of consumption are those who would have chosen this anyway, while others just modify their consumption towards the norm level.

${ }^{4}$ This is different from the question of whether if there is a single norm the precise level of consumption and the fraction of consumers adhering to the norm are unique.
} 
This has some striking implications for environmental policy in the case where the norm good generates damaging emissions. For now there are two externalities - the environmental externality and that generated by the desire to conform to the consumption decisions of others. Taken on its own we know that the environmental externality can be addressed by a variety of policies, particularly Pigovian tax policies that confront individuals with the social costs of their actions and so induces an efficient degree of mitigation whereby every individual reduces consumption of the polluting good by an amount that balances off the social harm with their individual attachment to the commodity. For this reason economists typically do not favour injunctive quantity-based policies that impose outright bans or uniform limits on the consumption of harmful commodities. ${ }^{5}$ However, as we will show, the presence of conformity considerations significantly affects the relative effectiveness and desirability of different forms of environmental policy, because, for the reasons explained above price-based policies may not cause consumers to reduce their consumption of the polluting good. In particular we show that: (i) the conventional optimum Pigovian tax (equal to marginal damage) may be ineffective in changing demand for the polluting good; (ii) while it is sometimes optimal to impose the conventional Pigovian tax equal to marginal damage, in other circumstances the optimal tax can be zero, or greater than marginal damage; (iii) in some circumstances an injunctive quantity-based policy may be preferable to a Pigovian tax.

The structure of the paper is as follows. In the next Section we give a brief summary of the extensive literature on norms and threshold behaviour, explaining more fully how our framework contributes and adds to it. In Section 3 we set out our model of consumption norms and analyse its implications for individual behaviour. In section 4 we determine what norms can emerge as stable equilibria. In section 5 we analyse the environmental policy implications of our model, establishing the results mentioned above. Section 6 concludes.

\section{Relation to the Literature}

As noted in the introduction, our model of conformity incorporates two related concepts from the broad literature on behavioural economics - consumption norms and threshold behaviour. We begin by setting out how our model relates to the existing literature on norms and threshold behaviour, and then discuss at greater length than in the introduction the two significant respects in which our model extends the existing literature.

\footnotetext{
${ }^{5}$ For example, prohibition on alcohol or tobacco consumption.
} 
By a consumption norm for a particular commodity we simply mean a particular level of consumption towards which people who wish to belong to a group adjust their consumption from the level they would have chosen if they were exercising only their self-regarding preferences - which for simplicity we define as their Marshallian levels of demand. Since our concept of a consumption norm arises simply from a desire to be seen to belong to a group of similar-minded individuals ${ }^{6}$, this can lead some individuals to reduce their consumption relative to their Marshallian levels of demand in order to conform with a particular norm.

We distinguish consumption norms from the broader concept of social norms. Social norms can be injunctive norms, where they relate to some underlying concept of moral behaviour, or, as with our consumption norms, they can be descriptive norms, where there is no underlying moral concept. We note two of the important roles commonly associated with social norms. First, as Young (2015) notes a key function of social norms is to coordinate people's expectations in interactions which are characterised by multiple equilibria, for example coordination games. Analysis of social norms often involves using evolutionary game theory to predict which of a multiplicity of possible outcomes emerge as stable equilibria and a focus on the design of punishment strategies by other players (e.g. Axelrod (1986)). ${ }^{7}$ Typically there are multiple stable equilibria, and these often involve discrete choices, such as whether or not to smoke in public (Nyborg and Rege (2003)) whether or not to recycle household waste (Brekke, Kipperberg and Nyborg (2010)).

A second aspect of social norms (dating back to Festinger (1954)) arises from people's uncertainty about their identity or opinions. For activities like provision of public goods, voting, or charitable giving, evidence suggests that individuals are more willing to contribute if they know members of their norm group have contributed or think others might match their contributions (referred to as conditional cooperation) - see for example Ledyard (1995), Azar (2004), Frey and Meier (2004), Tan and Bolle (2007), Gerber and Rogers (2009), Chaudhuri (2011), Bucholz, Falkinger and Rubbelke (2012), Abbott, Nandeibam and O'Shea (2013). Applying such concepts to the consumption of private goods, Hargreaves-Heap (2013) and Hargreaves-Heap and Zizzo (2009) identify a number of benefits from social

\footnotetext{
${ }^{6}$ The most influential sociological theories of consumption - especially Bourdieu's (1984) account of taste and distinction and Bauman's (1990) account of neo-tribal lifestyles - both present social norms and belonging as the fundamental mechanisms underpinning its contemporary social patterning (see Southerton (2002) for a full discussion). In our use of the term consumption norms should be interpreted as a subset of the much broader category of social norms which can affect behaviour.

${ }^{7}$ Axelrod's analysis also differs from ours in that he uses an evolutionary game approach, while we assume that individuals are conventional utility-maximisers, albeit with non-standard utility functions.
} 
norms, including (a) observing members of a norm group consuming a product an individual has not experienced can give implicit information about the quality of that product; (b) in a related manner, giving people information about what similar people achieve in saving energy, or retirement savings can significantly increase levels of savings (Allcott (2011) ${ }^{8}$; (c) by developing trust between members of a norm group, consumption norms can reduce transactions $\operatorname{costs}^{9}$; (d) for a number of consumption activities, such as reading a book or attending a concert, the benefits are not just the private experience but the subsequent opportunity to share thoughts about such experiences (the 'water cooler' effect) and this requires individuals to have overlapping sets of cultural interests.

In our analysis of consumption norms we assume that individuals are perfectly informed about the characteristics of products. Our concept of consumption norms is closer to that of Akerlof and Kranton (2000), who argued that an ability to identify with a group of people is a key part of self-identity and yields an important psychological benefit of belonging to a group, what Adam Smith referred to as the 'special pleasure of mutual sympathy' ${ }^{10}$. It is this pure psychological benefit of belonging to a group that we have in mind in this paper. An important implication is that it is the potential internal loss of such a benefit that provides the incentive for individuals to adhere to the consumption norm, rather than the use of punishment strategies by other players.

Our analysis of conformity shares some of the features in Bernheim (1994). In his model people differ in terms of their types (measured by a single index distributed over some interval). Society has a pre-specified notion of an ideal type and people suffer a loss of selfesteem the further their type is from the ideal. Individual's well-being depends on the utility they get from their actions, and the esteem in which they are held by others. If an individual's type was public information, all an individual could do is to act to maximise utility. But an individual's type is private information, and has to be inferred from one's actions, so individuals have an incentive to bias their actions towards that which an ideal person would perform; this leads some individuals to do more than they would do to maximise utility and

\footnotetext{
${ }^{8}$ See Bennett et al (2009) for a comprehensive analysis of the clustering of consumption activities based on overlapping cultural interests in the UK.

${ }^{9}$ This is linked to notions of social capital. It is important to distinguish between group membership developing greater trust between insiders - a positive social benefit - and developing a greater distrust of outsiders - a reduction in social benefit (see Putnam (2000) and Dasgupta (2000) for a recognition that social capital may have negative as well as positive effects). Hargreaves-Heap and Zizzo (2009) construct a measure to test this distinction, and in their experiments they find it is the negative effect which predominates.

${ }^{10}$ Hargreaves-Heap and Zizzo (2009) also develop a test to measure this psychological benefit of belonging to a group; they find that it balances out the negative effect of group membership noted in the previous footnote.
} 
others to do less. There are two possible equilibria: a fully-revealing equilibrium and a pooling equilibrium in which a group of individuals whose types are closer to the ideal type carry out the same level of action - so the equilibrium specifies a common action norm and the group of people who adhere to this common norm.

In our paper individuals differ in type in terms of their underlying Marshallian levels of demand for a good. Like Bernheim, we want to explain endogenously how wishing to adhere to a consumption norm changes an individual consumer's behaviour from its Marshallian level, which consumption norms can emerge as equilibrium norms, and how many equilibrium norms there might be. Unlike Bernheim we have no concept of an ideal type, and there is no private information - consumers are perfectly informed about the quality of the private goods they consume and all consumers know how many individuals are adhering to any given norm, which is the only information a consumer needs when deciding whether to adhere to a norm.

In our model an individual suffers a loss of well-being from not adjusting consumption away from her Marshallian level in order to adhere to a norm, but gains well-being from belonging to the group who also adhere to that norm; this benefit depends crucially on how many other individuals adhere to that norm. There is a critical threshold level of the number of people who have to adhere to a norm for an individual to derive a positive benefit from also adhering to that norm. In this respect our model draws on the literature on threshold effects, dating back to the pioneering work of Schelling (1971), Sakoda (1971), and Granovetter (1978). As Granovetter noted, it can be dangerous for social scientists, observing two otherwise similar populations with different behavioural outcomes, to infer that these differences reflect differences in underlying preferences - the populations may have very similar tastes but different thresholds.

Much of the literature on threshold effects follows Granovetter (1978) in assuming that the norm to which people might adhere involves a simple discrete choice - whether to adopt a new technology, whether or not to vote and, in the case of consumer demand, whether or not to buy a consumer durable (Granovetter and Soong (1986)). The key element of these models is that there is a distribution across the population of the threshold levels of other people making a particular choice before a particular individual makes the same choice. The analysis focuses on how different distribution functions affect the dynamic process by which individuals decide whether to adhere to a norm. Depending on this distribution function, the 
dynamic process can have multiple discrete equilibria, with some stable and others unstable. Granovetter and Soong (1986) show that this multiplicity of equilibria can have important implications in that once a system reaches a stable equilibrium it may require a very large shock to shift to another stable equilibrium, and this can lead to discontinuities in demand functions - below some price either no-one consumes the good or everyone consumes it which can have interesting implications for price-setting behaviour, particularly in imperfectly competitive markets ${ }^{11}$.

By contrast, we will assume that although the decision whether or not to adhere to a norm is discrete, the underlying norm is a continuous variable - the amount of a particular good an individual wishes to purchase - and there is continuous distribution of the underlying Marshallian demands in the population. More importantly, our model differs from models such as Granovetter and Soong (1986) in assuming that everyone has the same threshold in terms of the number of other individuals that need to adhere to the norm before an individual gets a positive benefit from adhering to the norm ${ }^{12}$.

A further important difference between our model and others in the literature is that they assume that an individual's utility loss in shifting consumption from the Marshallian demand towards a norm takes a simple quadratic form. This has the implication that people adjust their consumption towards the norm, but the only person who consumes at the norm level for that good is the individual whose Marshallian demand is the norm. This raises the question, often noted in the literature (e.g. Manski (2000)), that it can be difficult to identify the effect of consumption norms empirically. In contrast we assume that the utility loss suffered by an individual deviating from Marshallian demand depends on the absolute difference between the norm and an individual's Marshallian demand. As we will see this implies that if a norm exists, there will always be a potentially significant group of individuals, whose Marshallian demands lie in an interval around that norm who will consume the norm exactly if they decide to adhere to the norm. There may exist a second group of individuals, whose Marshallian demands are further from the norm than the first group, who adhere to the norm

\footnotetext{
${ }^{11}$ Granovetter and Soong (1986) also show if individuals have both lower thresholds and upper thresholds in their demand then there can there can be chaotic dynamic behaviour. Similarly complex dynamic processes can arise in models of opinion formation (see for example Hegselmann and Krause (2002), Buechel, Hellmann and Klossner (2015)).

${ }^{12}$ Allowing for individuals to differ in both their Marshallian demand levels and their threshold levels would be an important extension of this paper, though, as noted in the previous footnote this could potentially generate chaotic dynamic behaviour.
} 
by adjusting their consumption towards the norm (increasing their consumption if their Marshallian demand is below the norm, decreasing their consumption if their Marshallian demand is above the norm). Finally there may exist a third group whose Marshallian demands are even further from the norm who decide not to adhere to the norm and just consume their Marshallian demands. Indeed we will see that there are equilibria of our model where, although Marshallian demands vary systematically across the population, everyone consumes the norm level of consumption exactly. So there can be a striking difference between the pattern of consumption that would be observed with and without such a consumption norm.

Finally, we note that while this paper focuses on parameter values for which there will exist at least one stable equilibrium norm, we show that even if people do have a desire to adhere to a norm there are parameter values for which there is no stable equilibrium norm, and everyone consumes their Marshallian demands. This is similar to the point made by Fehr and Schmidt (2006) in their survey paper of the literature on another key area of behavioural economics- concerns for fairness, reciprocity and altruism. Fehr and Schmidt note that an important feature of recent developments in the empirical literature on experiments involving these features of behaviour is that they can explain why, under certain circumstances or parameter values (essentially those needed for perfectly competitive behaviour - e.g. large numbers of traders, perfect information about products) - even if people's preferences are not purely self-regarding the stable outcome of repeated experiments is consistent with the competitive equilibrium, while in markets with different parameter values - a few traders, informational frictions, or incomplete contracts - assuming that individuals behave as if they have self-regarding preferences does not predict the outcome well.

\section{A Model of Consumption Norms and Implications for Individual Behaviour}

\subsection{The Model}

There are two consumer goods, the individual consumption of which is denoted by the variables $x \geq 0, z \geq 0$, where $x$ is a commodity the level of whose consumption might be a norm ${ }^{13}$ and $z$ is expenditure on all other goods and serves as numeraire so its price is 1 . Good $x$ is produced in a perfectly competitive market with constant returns to scale, with price $p$ equal to the (constant) unit cost of production.

\footnotetext{
${ }^{13}$ In principle this could be an aggregate of goods which act as norms.
} 
We define a consumption norm by the pair $\left(x^{*}, n^{*}\right)$, where $x^{*}$ is the level of consumption towards which individuals may seek to adjust their consumption (adhere to that norm) and $n^{*}, 0 \leq n^{*} \leq 1$ is the fraction of the population adhering to that norm.

There is a population of individuals each of whom has income level $y$, and a utility function ${ }^{14}$

$$
\mathcal{P}\left(x, z, \delta ; x^{*}, n^{*}\right)=a x-\frac{x^{2}}{2}+z+\delta\left[\sigma\left(n^{*}\right)-\alpha\left|x^{*}-x\right|\right]
$$

where $\delta \in[0,1]$ is a choice variable that takes the value 1 if the individual chooses to adhere to a norm, and 0 if the individual chooses not to adhere and so behaves in a traditional Marshallian fashion. By substituting in the budget constraint, we can express utility in terms of the norm parameters $\left(x^{*}, n^{*}\right)$ and the two individual decision variables $-\delta$ and $x$ - as

$$
u\left(x, \delta ; x^{*}, n^{*}\right)=y+a x-p x-\frac{x^{2}}{2}+\delta\left[\sigma\left(n^{*}\right)-\alpha\left|x^{*}-x\right|\right]
$$

For an individual who has chosen to adhere to the norm we define $\alpha\left|x^{*}-x\right|$ as the strength of attraction of the norm level of consumption - the rate at which utility falls as individual consumption of $x$ deviates in either direction from the norm level. We use the absolute deviation rather than the more conventional square of the deviation, since, as we will show, this implies that there will always be a mass of individuals who consume the norm level of consumption exactly, whereas under the alternative specification the only individuals who will choose exactly the norm are those whose Marshallian level of consumption would have been $x^{*}$.

We define $\sigma\left(n^{*}\right)$ as the strength of desire for conformity with a group that makes up a fraction $n^{*}$ of the population. We assume that

$$
\sigma\left(n^{*}\right)=-\chi+\varphi n^{*}
$$

where $\chi>0$ is a fixed cost (i.e. unrelated to the level of consumption) of adhering to a norm group, i.e. of giving up individuality; while $\varphi>0$ is the rate at which the strength of the

\footnotetext{
${ }^{14}$ We take a special form of utility function to simplify the subsequent analysis of policy implications. In Dasgupta, Southerton, Ulph and Ulph (2016) we showed that the analysis of section 3 carries over to a general form of utility function.
} 
desire to conform grows with the fraction of the population, $n^{*}$, that choose to conform to $x^{*}$. So the strength of the desire for conformity captures a tension between a psychic cost of giving up one's sense of individuality and a psychic benefit/comfort from being part of a larger group.

For an individual to adhere to any norm the strength of desire for conformity must be positive when the entire population adheres to it -i.e. when $n^{*}=1$ - so we assume that $\varphi>\chi>0$, and let $\underline{n}=\frac{\chi}{\varphi}, \quad 0<\underline{n}<1$ be the minimum fraction of the population that need to adhere to a norm for that norm to have a positive strength of desire for conformity. We can therefore rewrite (1) as:

$$
u\left(x, \delta ; x^{*}, n^{*}\right)=y+a x-p x-\frac{x^{2}}{2}+\delta\left[\varphi\left(n^{*}-\underline{n}\right)-\alpha\left|x^{*}-x\right|\right]
$$

We initially assume that $0.5<\underline{n}<1$, which rules out a multiplicity of co-existent norms to which individuals would have to consider adhering. In section 3.2 we weaken this restriction and consider under what conditions there may exist two equilibrium norms.

Finally we assume that the taste parameter, $a$, is uniformly distributed in the population on the interval $[\underline{a}, \bar{a}], \quad 0<p<\underline{a}<\bar{a} .{ }^{15}$ We denote by $\omega=0.5(\bar{a}-\underline{a})$ the width of the distribution of preferences, or the degree of diversity of preferences.

In the rest of this section we take as given the existence of some norm $\left(x^{*}, n^{*}\right)$ and define for that norm a two-stage Nash equilibrium in which at stage 1 each individual decides whether or not to adhere to this norm while in stage 2 the individual chooses her utility-maximising level of consumption conditional on the adherence decision. We solve these decisions backwards.

\footnotetext{
${ }^{15}$ Given the quasi-linear structure of preferences the utility maximising choices of $x$ are independent of income, and so the precise distribution of income, $y$, plays no role in our analysis. All we require is that its distribution is sufficiently positively correlated with that of $a$ such that for all individuals $y>p(a-p+\alpha)$, and so individuals always buy a positive amount of the numeraire good.
} 


\subsection{Consumption decisions}

\subsubsection{Marshallian Consumption}

If an individual has chosen not to adhere to a norm - $\delta=0$ - then from (3) the utilitymaximising choice of $x$ is:

$$
x^{0}=\underset{x \geq 0}{\operatorname{ArgMax}} u\left(x, 0, x^{*}, n^{*}\right) \equiv \underset{x \geq 0}{\operatorname{ArgMax}} a x-\frac{x^{2}}{2}-p x=a-p .
$$

Given our assumption about the distribution of $a$ we take these Marshallian consumptions, $x^{0}$ to be uniformly distributed on the interval $[\underline{a}-p, \bar{a}-p] \equiv[\underline{X}, \bar{X}], \quad 0<\underline{X}<\bar{X}$, with mean $\mu=\frac{\bar{X}+\underline{X}}{2}$, and $\omega=\frac{\bar{X}-\underline{X}}{2}=\frac{\bar{a}-\underline{a}}{2}$ the width of the spread of tastes in the population. $(\omega$ will be a crucial variable in what follows). For an individual of type $x^{0}$ who has chosen not to adhere to the norm, the level of indirect utility associated with their Marshallian consumption is:

$$
v_{0}\left(x_{0}, x^{*}, n^{*}\right)=\operatorname{Max}_{x \geq 0} u\left(x, 0, x^{*}, n^{*}\right) \equiv \operatorname{Max}_{x \geq} y+a x-\frac{x^{2}}{2}-p x=y+\frac{\left(x_{0}\right)^{2}}{2} .
$$

\subsubsection{Consumption of individuals who adhere to the norm}

If $\delta=1$ the individual's utility-maximising consumption is:

$$
\hat{x}\left(x^{*}, n^{*}\right)=\underset{x \geq 0}{\operatorname{ArgMax}} u\left(x, 1, x^{*}, n^{*}\right) \equiv \underset{x \geq 0}{\operatorname{ArgMax}} a x-\frac{x^{2}}{2}-p x-\alpha\left|x^{*}-x\right| .
$$

An individual of type $x^{0}$ adhering to a norm $\left(x^{*}, n^{*}\right)$ chooses consumption $\hat{x}\left(x^{0} ; x^{*}\right)$

$$
\hat{x}\left(x^{0}, x^{*}\right)=\left\{\begin{array}{l}
x^{0}-\alpha \Leftrightarrow \quad x^{*}<x^{0}-\alpha \\
x^{*} \Leftrightarrow \quad x^{0}-\alpha \leq x^{*} \leq x^{0}+\alpha \\
x^{0}+\alpha \Leftrightarrow \quad x^{*}>x^{0}+\alpha
\end{array}\right.
$$

An individual of type $x^{0}$ adhering to a norm $x^{*}$ chooses consumption to lie within her normconsistent interval of consumption $\left[x^{0}-\alpha, x^{0}+\alpha\right]^{16}$ : (i) if the norm level of consumption is

\footnotetext{
${ }^{16}$ This illustrates what we call the gravitational pull of a consumption norm.
} 
sufficiently close to an individual's Marshallian level of consumption - specifically if it lies inside the individual's norm-consistent interval of consumption - the individual will consume the norm level exactly; (ii) if the norm level of consumption is outside an individual's normconsistent interval of consumption then the individual's consumption will lie at the boundary of the norm-consistent interval of consumption that is closest to the norm level ${ }^{17}$.

The indirect utility of an individual of type $x^{0}$ who adheres to the norm $\left(x^{*}, n^{*}\right)$ is

$$
v_{1}\left(x^{0}, x^{*}, n^{*}\right)=y+\varphi\left(n^{*}-\underline{n}\right)+\left\{\begin{array}{l}
\frac{\left(x^{0}-\alpha\right)^{2}}{2}+\alpha x^{*}, \quad x^{*}<x^{0}-\alpha \\
x^{0} x^{*}-\frac{\left(x^{*}\right)^{2}}{2}, \quad x^{0}-\alpha \leq x^{*} \leq x^{0}+\alpha \\
\frac{\left(x^{0}+\alpha\right)^{2}}{2}-\alpha x^{*}, \quad x^{*}>x^{0}+\alpha
\end{array}\right.
$$

\subsection{The decision to adhere to a norm}

The net benefit to an individual of type $x_{0}$ from choosing to adhere to the norm $\left(x^{*}, n^{*}\right)$ is

$$
\beta\left(x^{0}, x^{*}, n^{*}\right)=v_{1}\left(x^{0}, x^{*}, n^{*}\right)-v_{0}\left(x^{0}, x^{*}, n^{*}\right)
$$

Substitute in (5) and (8) to get

$$
\beta\left(x^{0}, x^{*}, n^{*}\right)=\varphi\left(n^{*}-\underline{n}\right)-L\left(x^{0}, x^{*}\right),
$$

where:

$$
L\left(x^{*}, x^{0}\right)=\left\{\begin{array}{l}
-\frac{\alpha^{2}}{2}+\alpha\left|x^{0}-x^{*}\right|, \quad\left|x^{0}-x^{*}\right|>\alpha \\
\frac{\left|x^{0}-x^{*}\right|^{2}}{2}, \quad\left|x^{0}-x^{*}\right| \leq \alpha
\end{array}\right.
$$

\footnotetext{
17 So there will be a range of individuals whose Marshallian demands differ from the norm who choose to consume exactly the consumption level specified by the norm. It is this clumping of demand that in our model makes the adherence to a norm distinct from a just a more concentrated distribution of preferences.
} 
captures the utility loss from adhering to a norm arising from two factors: (i) consumption is potentially different from the Marshallian amount; (ii) chosen consumption may also be different from the norm. For all individuals this loss is non-negative and is zero only for an individual whose Marshallian demand coincides with the consumption norm. The way this loss varies across individuals of different types is illustrated in Figure $1^{18}$.

Figure 1 here

It follows that an individual will adhere to a norm $\left(x^{*}, n^{*}\right)$ iff the net benefit from doing so is positive. This certainly requires that the fraction of consumers adhering to it is greater than the minimum threshold $\underline{n}-$ i.e. it requires $\beta\left(x^{0}, x^{*}, n^{*}\right)>0 \Rightarrow n^{*}>\underline{n}$;

Finally, for a given norm $\left(x^{*}, n^{*}\right)$, the range of types $x^{0}$ who would adhere to that norm, i.e. for whom, $\beta\left(x^{0}, x^{*}, n^{*}\right) \geq 0$ is given by $\left[\underline{x}^{0}\left(x^{*}, n^{*}\right), \bar{x}^{0}\left(x^{*}, n^{*}\right)\right]$, ignoring for the moment the need for $\underline{x}^{0}\left(x^{*}, n^{*}\right), \bar{x}^{0}\left(x^{*}, n^{*}\right)$ to lie in the range $[\underline{X}, \bar{X}]$. There are 2 cases, which are differentiated by whether the gains from adhering to a norm $x^{*}$ are greater or less than the costs of adhering to the norm when $x^{0}=x^{*} \pm \alpha$.

Case $A: \varphi\left(n^{*}-\underline{n}\right) \leq 0.5 \alpha^{2} \quad$ (a sufficient condition for this case to arise is $\varphi(1-\underline{n}) \leq 0.5 \alpha^{2}$ )

$$
\begin{aligned}
& \underline{x}^{0}\left(x^{*}, n^{*}\right)=x^{*}-\sqrt{2 \varphi\left(n^{*}-\underline{n}\right)} \geq x^{*}-\alpha ; \quad \bar{x}^{0}\left(x^{*}, n^{*}\right)=x^{*}+\sqrt{2 \varphi\left(n^{*}-\underline{n}\right)} \leq x^{*}+\alpha \\
& x^{0}<\underline{x}^{0}\left(x^{*}, n^{*}\right) \Rightarrow \hat{x}\left(x^{0}, x^{*}\right)=x^{0} \\
& \underline{x}^{0}\left(x^{*}, n^{*}\right) \leq x^{0}<\bar{x}^{0}\left(x^{*}, n^{*}\right) \Rightarrow \hat{x}\left(x^{0}, x^{*}\right)=x^{*} \\
& x^{0}>\bar{x}^{0}\left(x^{*}, n^{*}\right) \Rightarrow \hat{x}\left(x^{0}, x^{*}\right)=x^{0}
\end{aligned}
$$

See Figures 2A and 3A: (Figures 2A and 3A here)

Case $B: \varphi(n *-\underline{n})>0.5 \alpha^{2}$

$$
\underline{x}^{0}\left(x^{*}, n^{*}\right)=x^{*}-\frac{\alpha}{2}-\frac{\varphi}{\alpha}\left(n^{*}-\underline{n}\right)<x^{*}-\alpha ; \quad \bar{x}^{0}\left(x^{*}, n^{*}\right)=x^{*}+\frac{\alpha}{2}+\frac{\varphi}{\alpha}\left(n^{*}-\underline{n}\right)>x^{*}+\alpha
$$

18 The figures are at the end of this paper. 


$$
\begin{aligned}
& x^{0}<\underline{x}^{0}\left(x^{*}, n^{*}\right) \Rightarrow \hat{x}\left(x^{0}, x^{*}\right)=x^{0} \\
& \underline{x}^{0}\left(x^{*}, n^{*}\right) \leq x^{0}<x *-\alpha \Rightarrow \hat{x}\left(x^{0}, x^{*}\right)=x^{0}+\alpha \\
& x^{*}-\alpha \leq x^{0} \leq x^{*}+\alpha \Rightarrow \hat{x}\left(x^{0}, x^{*}\right)=x^{*} \\
& x^{*}+\alpha<x^{0} \leq \bar{x}^{0}\left(x^{*}, n^{*}\right) \Rightarrow \hat{x}\left(x^{0}, x^{*}\right)=x^{0}-\alpha \\
& x^{0}>\bar{x}^{0}\left(x^{*}, n^{*}\right) \Rightarrow \hat{x}\left(x^{0}, x^{*}\right)=x^{0}
\end{aligned}
$$

See Figures 2B and 3B: (Figures 2B and 3B here)

For both Case A and Case B we now take account of the need for $\hat{x}\left(x^{*}, x^{0}\right)$ to satisfy the condition: $\underline{X} \leq \hat{x}\left(x^{*}, x^{0}\right) \leq \bar{X}$. So define:

$$
\underline{\underline{x}}^{0}\left(x^{*}, n^{*}\right) \equiv \max \left[\underline{X}, \underline{x}^{0}\left(x^{*}, n^{*}\right)\right], \quad \overline{\bar{x}}^{0}\left(x^{*}, n^{*}\right) \equiv \min \left[\bar{X}, \bar{x}^{0}\left(x^{*}, n^{*}\right)\right]
$$

Then the two conditions a norm $\left(x^{*}, n^{*}\right)$ must satisfy to be a Nash equilibrium norm are ${ }^{19}$ :

$$
\begin{aligned}
& x^{*}=\left[\int_{\underline{\underline{x^{0}}}\left(x^{*}, n^{*}\right)}^{\overline{\bar{x}}^{0}\left(x^{*}, n^{*}\right)} \hat{x}\left(x^{0}, x^{*}\right) d x^{0}\right] /\left[\overline{\bar{x}}^{0}\left(x^{*}, n^{*}\right)-\underline{\underline{x}}^{0}\left(x^{*}, n^{*}\right)\right] \\
& n^{*}=\left[\overline{\bar{x}}^{0}\left(x^{*}, n^{*}\right)-\underline{\underline{x}}^{0}\left(x^{*}, n^{*}\right)\right] / 2 \omega \geq \underline{n} \geq 0.5
\end{aligned}
$$

Condition (15) is just the requirement that average consumption of those adhering to a norm equals the norm (where in constructing the average we need to scale the density function to reflect the fact that we are taking the average over the range of values $\left[\underline{x}^{0}\left(x^{*}, n^{*}\right), \overline{\bar{x}}^{0}\left(x^{*}, n^{*}\right)\right]$, which may be a strict subset of the range $[\underline{X}, \bar{X}])$. Condition (16) is just the requirement that the fraction of the population adhering to the norm must be $n^{*}$, and greater than $\underline{n}$.

\section{Existence of Equilibrium Norms}

We fix $\mu$, the mean value of $x^{0}$ and in section 4.1 we assume that $\underline{n}>0.5$ and investigate whether for any parameters $(\alpha, \varphi, \underline{n}, \omega)$ there exists a single equilibrium norm, and, if so, whether there is a unique value for a single equilibrium norm or there is a range of possible values a single equilibrium norm might take. In section 4.2 we relax somewhat the assumption that $0.5<\underline{n}$ and consider whether there might exist two equilibrium norms.

\footnotetext{
${ }^{19}$ In this Nash equilibrium individuals take $\left(x^{*}, n^{*}\right)$ as given. Qin and Janus (2018) consider a model of social norms where there is a small number of agents; each recognises that because the norm is the average value of actions they take, any individual's chosen action can influence the behaviour of others through the impact of on the social norm.
} 


\section{$\underline{4.1 \text { Existence of a Single Equilibrium Norm }}$}

We assume that $\underline{n}>0.5$. In Section 3.3 we analysed the decision to adhere to a norm for 2 cases $\mathrm{A}$ and $\mathrm{B}$ which depended on whether $\varphi\left(n^{*}-\underline{n}\right) \leq 0.5 \alpha^{2}$ or $\varphi\left(n^{*}-\underline{n}\right)>0.5 \alpha^{2}$ respectively. Since $n^{*}$ is an endogenous variable, we now define Cases A and B depending, respectively, on whether $\varphi(1-\underline{n}) \leq 0.5 \alpha^{2}$ or $\varphi(1-\underline{n})>0.5 \alpha^{2}$. In this section we analyse what are the single equilibrium norms for Cases A and B in sub-sections 4.1.1 and 4.1.2 respectively. To provide the intuition for the results we consider a particular set of parameters $(\alpha, \varphi, \underline{n})$ lying within some range and ask for, any value of $\omega$, what values of $x^{*}(\omega)$ with an associated value of $n^{*}\left(x^{*}(\omega), \omega\right)$ might $\left[x^{*}(\omega), n^{*}\left(x^{*}(\omega), \omega\right)\right]$ be an equilibrium norm. We do this in 3 stages; (i) for a given value of $\omega$ we ask if $[\mu, n *(\mu, \omega)]$ can be an equilibrium norm; (ii) then we ask, for that given value of $\omega$, for what interval of values of $x^{*}(\omega)$ around $\mu$ with associated values of $n^{*}\left(x^{*}(\omega), \omega\right)$ can $\left[x^{*}(\omega), n^{*}\left(x^{*}(\omega), \omega\right)\right]$ be a norm; we call this interval the 'wiggle room' around $\mu$ within which $\left[x^{*}(\omega), n^{*}\left(x^{*}(\omega), \omega\right)\right]$ can be a norm; (iii) finally we consider what happens to as $\omega$ varies, and show that as $\omega$ increases the 'wiggle room' shrinks until there is no equilibrium norm.

\section{$\underline{4.1 .1}$ Case A: $\varphi(1-\underline{n}) \leq 0.5 \alpha^{2}$}

We define $\xi(\omega) \equiv \sqrt{2 \varphi(1-\underline{n})}-\omega$ as the relevant wiggle room for this case. Then we have $\mathrm{e}^{20}$ :

Result 1 Case A. $\varphi(1-\underline{n}) \leq 0.5 \alpha^{2}$.

(i) If $\omega<2 \omega \leq \sqrt{2 \varphi(1-\underline{n})}<\alpha$ then for any $x^{*} \in[\underline{X}, \bar{X}],\left(x^{*}, 1\right)$ is an equilibrium norm to which everyone adheres exactly.

(ii) If $\omega<\sqrt{2 \varphi(1-\underline{n})}<\min (\alpha, 2 \omega)$, then for any $x^{*} \in[\mu-\xi(\omega), \mu+\xi(\omega)],\left(x^{*}, 1\right)$ is an equilibrium norm to which everyone adheres exactly; as $\omega$ increases $\xi$ falls.

(iii) If $\omega=\sqrt{2 \varphi(1-\underline{n})}<\min (\alpha, 2 \omega)$ then $(\mu, 1)$ is the unique equilibrium norm to which everyone adheres exactly.

(iv) If $\sqrt{2 \varphi(1-\underline{n})}<\omega<\alpha$ then there is no equilibrium norm.

${ }^{20}$ All proofs are in the Appendix. 
The situation is shown in Figure 4. It is clear that $\xi(\omega)$ is a decreasing function of $\omega$. For the parameters in Result 1(i) $\xi(\omega) \geq \omega$, the wiggle room is at least as large as the width of the interval, so for any $x^{*},\left(x^{*}, 1\right)$ is an equilibrium norm. Results 1(ii)-(iv) show that as $\omega$ increases the wiggle room steadily decreases until only $(\mu, 1)$ can be a stable equilibrium, and for any higher value of $\omega$ there cannot be a stable equilibrium.

It is important to note that in Results 1(i) -(iii) those who adhere to the norm do so exactly. The intuition is that given the limit on the size of $\omega$, it would not be possible to have an equilibrium norm $\left(x^{*}, 1\right)$ to which some people adhered exactly and some adhered by adjusting actual demands either up or down by an amount $\alpha$, because then the average consumption of those adhering to the norm would not be equal to the norm, violating condition (15).

The intuition behind Result 1(iv) is that for these parameter values, there are people at the extremes of consumption for whom the costs of adhering to the norm $(\mu, 1), 0.5 \omega^{2}$, exceed the benefit of adhering to the norm $(\mu, 1), \varphi(1-\underline{n})$, that would arise if everyone adhered to that norm. So these individuals do not adhere, implying that $n^{*}$ would now be $<1$. But this causes the benefit of adhering to the norm to fall to $\varphi\left(n^{*}-\underline{n}\right)$, so again there are marginal people for whom costs exceed benefits, and $n^{*}$ shrinks. This continues until $n^{*}<\underline{n}$. So when $\varphi(1-\underline{n})<0.5 \omega^{2} \leq 0.5 \alpha^{2}$ there is no equilibrium norm.

\subsubsection{Case B (i): $\varphi(1-\underline{n})>0.5 \alpha^{2} \geq 0.5 \omega^{2}$}

We start with the subset of parameter values for Case B where $\varphi(1-\underline{n})>0.5 \alpha^{2}$ and $\omega \leq \alpha$. Then the situation is essentially equivalent to Result 1 , but with wiggle room $\psi(\omega) \equiv \alpha-\omega$. So we have:

Result 2: $\varphi(1-\underline{n})>0.5 \alpha^{2} \geq 0.5 \omega^{2}$.

(i) If $\omega \leq 0.5 \alpha$ then for any $x^{*} \in[\underline{X}, \bar{X}],\left(x^{*}, 1\right)$ is an equilibrium norm to which everyone adheres exactly.

(ii) If $0.5 \alpha<\omega<\alpha$, then for any $x^{*} \in[\mu-\psi(\omega), \mu+\psi(\omega)],\left(x^{*}, 1\right)$ is an equilibrium norm to which everyone adheres exactly; as $\omega$ increases $\psi(\omega)$ falls.

(iii) If $\omega=\alpha$ then $(\mu, 1)$ is the unique equilibrium norm to which everyone adheres exactly. 
The Result is shown Fig. 5.

\section{$\underline{4.1 .3 \text { Case B(ii): }} \varphi(1-\underline{n}) \geq-0.5 \alpha^{2}+\alpha \omega>0.5 \alpha^{2}$}

Define $\bar{\omega} \equiv \frac{\varphi(1-\underline{n})}{\alpha}+0.5 \alpha$ as the value of $\omega$ for which the loss to the marginal person adhering to the norm just equals the gain to adhering to the norm when everyone adheres. Also define $n^{*}[x *(\omega), \omega]=n^{*}(\omega) \equiv \frac{\varphi \underline{n}-0.5 \alpha^{2}}{\varphi-\alpha \omega}$ as the value of $n^{*}(\omega)$ for which the benefit to the marginal person adhering to a norm to which $n^{*}(\omega)$ already adhere, $\phi\left(n^{*}(\omega)-\underline{n}\right)$, just equals the loss of utility from adhering to that norm $-0.5 \alpha^{2}+\alpha n^{*}(\omega) \omega$. Note that $n^{*}(\omega)$ does not depend on $x *(\omega)$. Finally we define the relevant 'wiggle room' as $\eta(\omega)=\mu+\omega-\mu-n^{*}(\omega) \omega=\omega\left(1-n^{*}(\omega)\right)=\omega\left[\frac{\varphi(1-\underline{n})-\left(\alpha \omega-0.5 \alpha^{2}\right)}{\varphi-\alpha \omega}\right]$. Then we have:

Result 3: $\varphi(1-\underline{n}) \geq-0.5 \alpha^{2}+\alpha \omega>0.5 \alpha^{2}$

(i) For $\alpha<\omega<\bar{\omega}$ there are two possible equilibrium norms: $(\mu, 1)$ and $\left(x^{*}, n^{*}(\omega)\right)$ where $x^{*} \in[\mu-\eta(\omega), \mu+\eta(\omega)]$.

(ii) For $\omega=\bar{\omega}$, the only equilibrium norm is $(\mu, 1)$.

(iii) For $\omega>\bar{\omega}$ there is no equilibrium norm.

(iv) In cases (i) and (ii) individuals with Marshallian demands $x^{0} \in\left[x^{*}-\alpha, x^{*}+\alpha\right]$ adhere exactly to the norm $x *$; individuals with Marshallian demands $x^{0} \in\left[x^{*}-n^{*}(\omega) \omega, x^{*}-\alpha\right]$ adhere by setting $\hat{x}=x^{0}+\alpha ;$ individuals with Marshallian demands $x^{0} \in\left[x^{*}+\alpha, x^{*}+n^{*}(\omega) \omega\right]$ adhere by setting $\hat{x}=x^{0}-\alpha$.

The result is shown in Figures 6, 7 and 8.

There are two significant features of Result 3 (i) which relate back to the discussion of threshold dynamics in the Introduction. First, for any value of $\omega$ there are two distinct types of equilibrium norms: $(\mu, 1)$ to which everyone adheres, and a range of values of $x *$ to which only a fraction $n^{*}(\omega)$ of the population adhere.

Second, the first type of equilibrium norm $(\mu, 1)$ is unstable in the sense that there is no other value of $x^{*}$ for which $\left(x^{*}, 1\right)$ can be an equilibrium norm. The rationale is straightforward see Figure 7 . In the case of the norm $(\mu, 1)$ people with Marshallian demands 
$x^{0} \in[\mu-\alpha, \mu+\alpha]$ adhere exactly; those with Marshallian demands $x^{0} \in[\mu-\omega, \mu-\alpha]$ consume $\hat{x}=x^{0}+\alpha$; those with Marshallian demands $x^{0} \in[\mu+\alpha, \mu+\omega]$ consume $\hat{x}=x^{0}-\alpha$. So the average level of consumption of those adhering to $(\mu, 1)$ is equal to $\mu$. To see why, $\left(x^{*}\right.$, 1), $x^{*} \neq \mu$ could not be an equilibrium norm suppose $x^{*}>\mu$; then the set of people with demands $x^{0}>x^{*}+\alpha$ adhering to the norm by setting $\hat{x}\left(x^{0}, x^{*}\right)=x^{0}-\alpha$ will be smaller than the set of people with demands $x^{0}<x^{*}-\alpha$ adhering to the norm by setting $\hat{x}\left(x^{0}, x^{*}\right)=x^{0}+\alpha$. Thus average consumption of those adhering to the norm does not equal the norm, and so ( $x^{*}$, 1) cannot be an equilibrium norm. A similar argument applies if $x^{*}<\mu$.

Finally, for results (ii) and (iii) it is straightforward to see that $n *(\omega)$ is an increasing function of $\quad \omega, \quad$ with $\quad n^{*}(\alpha)=\frac{\varphi \underline{n}-0.5 \alpha^{2}}{\varphi-\alpha^{2}} \Rightarrow \quad \underline{n}<n^{*}(\alpha)<1 \quad$ and $\quad n^{*}(\bar{\omega})=1 . \quad$ Hence: $\eta(\alpha)=\alpha\left(1-n^{*}(\alpha)\right)>0$ and $\eta(\bar{\omega})=0$. This suggests that the wiggle room $\eta(\omega)$ shrinks as $\omega$ increases, and that when $\omega=\bar{\omega}$ the only equilibrium norm is $(\mu, 1)$ (see Figure 8). However we have not been able to establish that $\eta(\omega)$ shrinks monotonically to zero.

This completes our analysis of possible $\underline{n}>0.5$ single equilibrium norms for all possible values of the parameters $(\alpha, \varphi, \underline{n}, \omega)$.

\section{$\underline{4.2 \text { Two Equilibrium Norms }}$}

We now relax the assumption that $\underline{n}>0.5$ so that we have the possibility that more than one equilibrium norm could co-exist; so we now assume that $0.5>\underline{n}>1 / 3$, which is a necessary condition to have two equilibrium norms.

We now consider what further conditions the parameters $(\alpha, \varphi, \underline{n}, \omega)$ need to satisfy to allow for two equilibrium norms. First we will use Result 3 to ensure that we can have equilibrium norms of the form $\left(x^{*}, n^{*}(\omega)\right)$. This requires the following condition

$$
\varphi(1-\underline{n})>-0.5 \alpha^{2}+\alpha \omega>0.5 \alpha^{2} \Leftrightarrow \frac{\varphi(1-\underline{n})}{\alpha}+0.5 \alpha>\omega>\alpha
$$

More importantly to have two equilibrium norms we need to also ensure that $0.5>n *(\omega)>\underline{n}>1 / 3$. This can be shown to require that parameters $(\alpha, \varphi, \underline{n}, \omega)$ satisfy two 
further conditions, namely: $0.5 \alpha+\varphi(1-\underline{n}) / \alpha>\omega>\max [1.5 \alpha, \alpha+\varphi(1-2 \underline{n}) / \alpha] \quad$ and $\varphi \geq 1.5 \alpha^{2} \Rightarrow \underline{n}>1 / 3 \geq 0.5 \alpha^{2} / \varphi$.

It is intuitively clear that if $x *$ is located around the median, $\mu$, there will not be enough space to allow for two equilibrium norms. This is demonstrated in Result 4 below.

Result 4: Suppose (a) $0.5 \alpha+\varphi(1-\underline{n}) / \alpha>\omega>\max [1.5 \alpha, \alpha+\varphi(1-2 \underline{n}) / \alpha]$ and (b) $\varphi \geq 1.5 \alpha^{2} \Rightarrow \underline{n}>1 / 3 \geq 0.5 \alpha^{2} / \varphi$ and define: $\rho(\omega)=n^{*}(\omega) \omega$. Then:

(i) $0.5>n *(\omega)>\underline{n}>1 / 3$

(ii) If $\mu+\omega-3 \rho(\omega)<x^{*}<\mu-\omega+3 \rho(\omega)$ then $\left(x^{*}, n^{*}(\omega)\right)$ is the unique equilibrium norm.

(iii) If $\mu-\omega<x^{*}<\mu+\omega-3 \rho(\omega) \Rightarrow \mu+\omega-\left(x^{*}+\rho(\omega)\right)>2 \rho(\omega) \quad-\quad$ so there is sufficient room for a second norm $\left(x^{* *}, n(\omega)\right)$ with $x^{* *}>x^{*}$; similarly if $\mu+\omega>x^{*}>\mu-\omega+3 \rho(\omega) \Rightarrow\left(x^{*}-\rho(\omega)\right)-(\mu-\omega)>2 \rho(\omega) \quad-\quad$ so there is sufficient room for a second norm $\left(x^{* *}, n(\omega)\right)$ with $x^{* *}<x^{*}$.

The intuition is that if $\left(x^{*}, n^{*}(\omega)\right)$ exists such that $1 / 3<\underline{n}<n^{*}(\omega)<0.5$, then it is clear that we can have 1 or 2 equilibrium norms depending on the value of $x^{*}$. If $x^{*}$ lies in an interval around $\mu$ as defined in (ii) there is insufficient space in the remaining tails of the interval for a second norm. However if $x^{*}$ lies in an interval bounded respectively below (above) by $\underline{X}(\bar{X})$, as defined in (iii), then there is sufficient space for a second norm of $x^{* *}$ above (below) $x *$ for a second norm $\left(x^{* *}, n^{*}(\omega)\right)$.

Since individuals will only adhere to a norm if they are at least as well off as they would be consuming their Marshallian demands then wellbeing must be higher with two equilibrium norms than with one equilibrium norm. Of course this conclusion depends strongly on our assumption of a uniform distribution of preferences.

This completes our analysis of what consumption norms can be considered equilibrium norms.

\section{Policy Implications}

In this section we consider the policy implications of our analysis of consumption norms particularly in the case when the commodity that is a norm is also the source of 
environmentally damaging emissions. As noted in the introduction there are two externalities: that which arises from the interdependence of consumption decisions when individuals have a preference for conformity; and the traditional environmental externality. To fully understand the implications of having both externalities, in Section 5.1 we briefly consider the policy implications when there is no environmental externality and then, in Section 5.2 we introduce the environmental externality, briefly consider the classical prescriptions for environmental policy and then consider the optimal environmental and other policies when both externalities are present.

\subsection{Only the Consumption Norm Externality}

In this sub-section we assume again that $0.5<\underline{n}<1.0$ so only one equilibrium norm $\left(x^{*}, n^{*}\right)$ can exist, though there can be a range of possible values for that single norm. Because there may be multiple possible values for an equilibrium norm, and because individuals simply take such a norm as given, we ask whether from a welfare perspective there is an optimal consumption norm and if so what policies might bring such an optimal norm about.

In Results 1, 2 and 3 we have shown that, under appropriate parameter values, there are ranges of possible values of $x^{*}$ for which a single equilibrium norm, $\left(x^{*}, n^{*}\right)$ exists, where the associated value for $n^{*}$ is either 1 or the constant $\frac{\varphi \underline{n}-0.5 \alpha^{2}}{(\varphi-\alpha \omega)}$ independent of $x^{*}$. We now consider the following thought experiment: if a policy maker is able to choose one of these values for $x^{*}$, which one would be chosen? From (14) the net benefit to an individual with Marshallian demand $x^{0}$ of adhering to a norm $x^{*}$ is given by $\varphi\left(n^{*}-\underline{n}\right)-L\left(x^{0}, x^{*}\right)$, where $L\left(x^{0}, x^{*}\right)$ is given by (13). So if we ask which value of $x^{*}$ maximises the expected net benefit of adhering to that norm, that is equivalent to choosing $x *$ to minimise the expected value of $L\left(x^{0}, x^{*}\right)$, denoted $\underset{x^{0}}{E}\left[L\left(x^{0}, x^{*}\right)\right]$.

We have the following result:

\section{Result 5.}

(i) In Results 1 and 2, where equilibrium norms take the form $\left(x^{*}, 1\right)$, the unique value of $x^{*}$ which minimises the expected value of the utility loss $\underset{x^{0}}{E}\left[L\left(x^{0}, x^{*}\right)\right]$ is $x^{*}=\mu$ 
(ii) In Result 3, where the equilibrium norm takes the form $\left(x^{*}, n^{*}(\omega)\right)$, there is no unique optimal value of $x^{*}$, since the value of $\underset{x^{0}}{E}\left[L\left(x^{0}, x^{*}\right)\right]$ is the same for all possible equilibrium values of $x^{*}$.

The intuition behind these results is that in Results 1 and 2, because everyone adheres to a norm, if $x^{*} \neq \mu$ the loss of utility from those furthest from $x^{*}$ outweigh losses from those closer to the norm, so centring the norm on the mean value of Marshallian demand reduces these extreme losses. On the other hand in Result 3 because not everyone adheres to the norm the losses of those adhering to the norm are the same no matter which value of $x^{*}$ constitutes the norm $\left(x^{*}, n^{*}(\omega)\right)$.

What does Result 5 imply for policy? It is inherent in our analysis underlying Result 5 that, although we focus the existence of a single equilibrium norms, $\left(x^{*}, n^{*}\right)$, if there is a range of values that $x^{*}$ might take we have not analysed the process by which a particular value of $x^{*}$ emerges as an equilibrium norm. So we assume that policy makers are not able to directly manipulate the value of $x^{*}$. But we assume that policy makers are able to use taxes and lumpsum transfers to shift the Marshallian demand for the norm good. In Results 1 and 2 expected utility is maximised when Marshallian demand of an individual with the mean value of the taste parameter for the norm good is equal to the norm. So if $x^{*}<\mu$ the optimal policy is to tax the norm good to reduce mean demand to the norm, while if $x^{*}>\mu$ the optimal policy is to subsidise the norm good to raise mean demand to the norm. In Result 3 there is no role for policy.

We stress that these policy conclusions that arise when there are consumption norms are quite different from those emerging from models without consumption norms. For example, in a standard model of purely self-interested individuals where, as in our model, utility is linear in income, there is no role for corrective taxation, whereas in our model we predict the use of either a consumption tax, consumption subsidy or no corrective taxation. Of course the role for taxation in our model arises because the introduction of a consumption norm generates a form of consumption externality. By contrast, in models where there is conspicuous consumption (Veblen effect), there is also an externality - since individuals care about their own consumption relative to that of some group - but here the standard policy prescription is a tax on either consumption (Layard, 2006), or labour (Slack and Ulph, 2016), whereas, in our framework the policy could involve subsidising consumption. So again the policy prescriptions from our model differ from the conventional one. 


\subsection{Consumption Norm Externality and Environmental Externality}

We now suppose that each unit of consumption of the norm good generates a unit of environmental damage with a constant unit damage cost $\gamma$, and the only way of reducing this environmental damage is to reduce the consumption of the norm good. As in Section 5.1 we suppose that parameter values are such that, prior to any environmental policy, in Results 1, 2 and 3 , there is a single equilibrium norm, $\left(x^{*}, n^{*}\right)$, where $x^{*}$ lies in an interval $\left[\underline{\zeta}^{0}, \bar{\zeta}^{0}\right]$ where the values $\underline{\zeta}^{0}, \bar{\zeta}^{0}$ depend on the parameter values determining which of the Results 1,2 or 3 apply, and $n^{*}$ takes the value 1 in Results 1 and 2 and the value $\frac{\varphi \underline{n}-0.5 \alpha^{2}}{(\varphi-\alpha \omega)}$ in Result 3. Prior to any environmental policy being implemented, aggregate emissions are denoted by $\Sigma\left(x^{*}, n^{*}\right) \equiv\left\{\int_{\underline{X}}^{\bar{X}} \hat{x}\left(x^{0}, x^{*}\right) d x^{0}\right\} /(\bar{X}-\underline{X}) ;$ and the aggregate net benefit of adhering to $\left(x^{*}, n^{*}\right)$ are denoted by $B\left(x^{*}, n^{*}\right) \equiv\left\{\int_{\underline{\underline{x}}^{0}(\cdot)}^{\bar{x}^{0}(\cdot)}\left[\varphi\left(n^{*}-\underline{n}\right)-L\left(x^{0}, x^{*} n^{*}\right)\right] d x^{0}\right\} /\left[\overline{\bar{x}}^{0}\left(x^{*}, n^{*}\right)-\underline{\underline{x}}^{0}\left(x^{*}, n^{*}\right)\right]$.

To take account of environmental damage costs, individual well-being is now given by:

$$
u\left(x, \delta ; x^{*}, n^{*}, \Sigma\right)=y+a x-p x-0.5 x^{2}+\delta\left[\varphi\left(n^{*}-\underline{n}\right)-\alpha\left|x^{*}-x\right|\right]-\gamma \Sigma\left(x^{*}, n^{*}\right)
$$

We assume that each individual recognises that in this atomless economy her consumption decisions have no impact on aggregate consumption of the polluting good and so treats $\Sigma\left(x^{*}, n^{*}\right)$ as fixed. Hence the behavioural results of sections 3 and 4 are unaffected by the inclusion of the harm from environmental damages in individual utility.

We now consider possible policy options. Suppose first that the government imposes a Pigovian emission tax, $\tau$, and rebates the tax revenue raised as a lump-sum to each individual.

In the absence of a norm this will increase the price of the good by $\tau$ and thereby reduce by $\tau$ every consumer's Marshallian demand ${ }^{21}$, mean Marshallian demand, $\mu$, and the range of values, $[\mu-\omega, \mu+\omega]$, within which Marshallian demands will lie. From the standard theory

\footnotetext{
${ }^{21}$ The fact that every consumer reduces demand by the same amount is an artefact of our model which, from (1), assumes that the coefficient on the negative quadratic term is $1 / 2$ for every consumer. In a more general model individuals would cut back their demands in varying amounts depending on their degree of attachment to the good.
} 
of environmental economics we know that, in the absence of consumption norms, the firstbest optimal emission tax is $\tau=\gamma$ i.e. the emission tax equals marginal damage $\operatorname{cost}^{22}$.

However if there are consumption norms, then, in addition to the impact of the emission tax, $\tau$, on Marshallian demands, the pre-tax norm $\left(x^{*}, n^{*}\right)$ will remain an equilibrium norm so long as $x^{*} \leq \mu+\zeta-\tau \Leftrightarrow \tau \leq \zeta+x^{*}-\mu \equiv \bar{\tau} ; \quad \bar{\tau}$ is the minimum emission tax that needs to be set to induce individuals to shift from the norm, for all cases except the unstable norm $(\mu, 1)$ in Result 3, for which any non-zero emission tax will cause individuals to abandon the norm. $\bar{\tau}$ depends on the extent of wiggle room $\zeta$, and where $x^{*}$ lies relative to the average level of Marshallian consumption. $n^{*}$ is not affected by the emission tax.

Then it is straightforward to see that the effects of imposing an emission tax $\tau>0$ are as follows.

Result 6. Suppose that prior to any environmental policy there is an equilibrium norm $\left(x^{*}\right.$, $\left.n^{*}\right)$. The government then imposes an emission tax $\tau$.

(i) If the parameters $(\alpha, \varphi, \underline{n}, \omega)$ are such that Result 1 or Result 2 apply and $\tau \leq \bar{\tau}$, then all consumers continue to adhere to the norm $\left(x^{*}, 1\right)$; so the emission tax is completely ineffective. If $\tau>\bar{\tau}$ everyone reverts to their Marshallian consumption levels, reduced by $\tau$.

(ii) If parameters $(\alpha, \varphi, \underline{n}, \omega)$ are such that Result 3 applies and $\left(x^{*}, n^{*}\right)$ is the initial equilibrium norm, then if $\tau \leq \bar{\tau}$, a proportion $n^{*}$ of consumers continue to adhere to the norm $\left(x^{*}, n^{*}\right)^{23}$ while a proportion $\left(1-n^{*}\right)$ of consumers will reduce consumption by an amount $\tau$; so the emission tax is partially effective. If $\tau>\bar{\tau}$ everyone reverts to their Marshallian consumption levels, reduced by $\tau$.

(iii) If parameters $(\alpha, \varphi, \underline{n}, \omega)$ are such that Result 3 applies and $(\mu, 1)$ is the initial equilibrium norm, then for any $\tau>0$ everyone reverts to their Marshallian consumption levels, reduced by an amount $\tau$.

\footnotetext{
22 Notice that in general this will not reduce consumption and hence emissions to zero but just causes each individual to adjust their consumption by an amount that reflects the balance between the strength of their desire for the commodity and the social harm it causes.

${ }^{23}$ They will not be the same consumers as those adhering to the norm prior to the imposition of the emission tax.
} 
Result 6 just describes the effect of imposing an emission tax $\tau$. In cases 6(i) and 6(ii) if an emission tax is below a critical level, $\bar{\tau}$, it is either wholly or partially ineffective. In case 6(iii) any emission tax eliminates the norm so there is just one externality.

We now ask what is the optimal (second-best) emission tax? To address this question, policy makers need to take account of the average net benefit per person of adhering to the norm $\left(x^{*}, n^{*}\right)$, denoted by:

$$
B\left(x^{*}, n^{*}\right) \equiv\left\{\int_{\underline{x}^{0}(\cdot)}^{\overline{\bar{x}}^{0}(\cdot)}\left[\varphi\left(n^{*}-\underline{n}\right)-L\left(x^{0}, x^{*} n^{*}\right)\right] d x^{0}\right\} /\left[\overline{\bar{x}}^{0}\left(x^{*}, n^{*}\right)-\underline{\underline{x}}^{0}\left(x^{*}, n^{*}\right)\right]
$$

The optimal policy involves choosing between an outcome in which the relevant proportion, $n^{*} \leq 1$, of the population continue to adhere to the norm $\left(x^{*}, n^{*}\right)$ or switch to their Marshallian demands, which, except for Result 6(iii) ${ }^{24}$, requires setting an emission tax $\tau>\bar{\tau}$. Switching from the norm to Marshallian demands at some tax $\tau$ yields a net benefit $n^{*}\left[W^{P}(\tau)-B\left(x^{*}, n^{*}\right)\right]$, where $W^{P}(\tau) \equiv 0.5 \tau(2 \gamma-\tau)$ is the standard Pigovian net gain of reducing environmental damage by an amount $\gamma \tau$ net of the loss of consumer surplus $0.5 \tau^{2}$; $W^{P}(\tau)$ takes a maximum $0.5 \gamma^{2}$ when $\tau=\gamma$. So the existence of consumption norms imposes two constraints on the use of an emission tax: it requires the benefit of reducing emissions to exceed the benefit of adhering to a norm, and, except for the special case in Result 6(iii), it requires the emission tax to exceed a minimum level $\bar{\tau}$. Then it is straightforward to see that the optimal second-best emission tax is as follows:

\section{Result 7}

(i) Suppose parameter values are such that Result 3 applies and the pre-policy equilibrium norm is $(\mu, 1)$, then the optimal policy is to impose no emission tax if $B\left(x^{*}, n^{*}\right) \geq W^{P}(\gamma)$, and otherwise to impose the optimal (first best) Pigovian tax, $\tau=\gamma$.

(ii) Suppose parameter values are such that Results 1 and 2 apply with $\left(x^{*}, 1\right)$ the prepolicy equilibrium norm, while in Result $3\left(x^{*}, n^{*}\right)$ is the equilibrium norm with $n^{*}<1$. Suppose also that $\bar{\tau}<\gamma$. Then the optimal policy is to impose any

\footnotetext{
${ }^{24}$ There is an argument that even in Results 6(i) and 6(ii) the imposition of any emission tax could destroy the benefit of adhering to a norm by eliminating self-attribution (see, for example, Perino, Panzone and Swanson, 2014). We do not consider this effect.
} 
emission tax $\tau \leq \bar{\tau}$ if $B\left(x^{*}, n^{*}\right) \geq W^{P}(\gamma)$, and otherwise to impose the optimal (first best) Pigovian tax, $\tau=\gamma$.

(iii) Suppose parameter values are such that Results 1 and 2 apply with $\left(x^{*}, 1\right)$ the prepolicy equilibrium norm, while in Result $3\left(x^{*}, n^{*}\right)$ is the equilibrium norm with $n^{*}<1$. Suppose also that $\bar{\tau} \geq \gamma$. Then the optimal policy is to impose any emission tax $\tau<\bar{\tau}$ if $B\left(x^{*}, n^{*}\right) \geq W^{P}(\bar{\tau})$, and otherwise to impose the tax, $\bar{\tau}$.

Results 7(i) and (ii) are cases where the constraint on imposing the conventional optimal emission tax $\tau=\gamma$ is that the conventional benefits of imposing an emission tax equal to marginal damage costs must exceed the average benefits of adhering to the norm. In Result 7(iii) there is the additional constraint that the minimum level of tax needed to induce people to abandon their norm now exceeds the marginal damage cost, reducing the conventional benefit from implementing an emission tax, and hence making it more likely that the optimal policy is to leave the norm unaffected.

Now suppose environmental policy is 'quantity-based' rather than 'price-based', for example, mandating all individuals to reduce their consumption by an amount $\gamma$. Suppose that such an injunctive norm means that consumers would view this policy as losing the net benefit $B\left(x^{*}, n^{*}\right)$ of voluntarily conforming to the original norm $\left(x^{*}, n^{*}\right)$. Then, just as in Results 7(i) and (ii), there is still the constraint on imposing this quantity-based policy that the conventional benefits from implementing an environmental policy must exceed the benefits from adhering to the norm to justify this quantity-based policy. So for parameter values for which Results 7(i) and 7(ii) apply, there is an equivalence between the tax-based policy and the quantity-based policy. But, in comparison with Result 7(iii), when the quantity-based policy is employed there is no equivalent to the constraint in Result 7(iii) on the minimal level of tax required to shift consumers from adhering to the norm. So there are parameter values for which it would not be desirable to impose an emission tax but it would be desirable to use a quantity-based policy.

Obviously if consumers did not view the quantity-based policy as eliminating their benefits of adhering to the norm, then the optimal policy would be to simply mandate all consumers to reduce their consumption of this good by $\gamma$. A better way of thinking about this situation is to consider policies designed to shift norms. As noted earlier, it is perhaps easier to think about policies for shifting such norms when the norm involves a discrete choice - whether or not to 
smoke in public places (Nyborg and Rege (2003)) or whether or not to recycle some forms of household waste (Brekke, Kipperberg and Nyborg (2010)).

A related issue is whether the government might use monetary measures to either reduce the benefits $B\left(x^{*}, n^{*}\right)$ of adhering to a norm, or compensate them for foregoing such benefits ${ }^{25}$. But apart from the question of how such payments might be designed, the government would need to raise taxes to fund such payments - thereby reducing individual well-being by a different but possibly equivalent route.

Finally even if the optimal policy involves individuals no longer adhering to the original consumption norm $\left(x^{*}, n^{*}\right)$ it could be argued that this is a short-term effect and a new norm might emerge. We do analyse the dynamic process by which a new norm would emerge. But the speed with which the new norm emerges may depend on how close that norm is to the original norm. So suppose in the interval $[\mu-\tau-\zeta, \mu-\tau+\zeta]$ a new norm $\left(x^{* *}, n^{*}\right)$ would have $x^{* *}=\mu-\tau+\zeta$, i.e. a new norm is close as possible to the previous norm. Then the size of the emission tax $\tau$ might be reduced relative to the findings in Result 7 to ensure a speedier convergence to a new norm, and hence the reinstatement of the benefits of adhering to a norm. Clearly this is an area for future research.

\section{$\underline{\text { 6. Conclusions }}$}

In this paper we have presented a model of consumption norms in which, for some goods, there may exist a level of consumption to which individuals wish to conform because they benefit from being identified as belonging to a group of like-minded individuals. Unlike the Veblen notion of conspicuous consumption this desire for conformity will lead some individuals to reduce their consumption from the Marshallian level to the norm. Our modelling of the welfare cost of adhering to the norm also has the important implication that a significant number of people, in some cases the entire population, will consume the norm exactly. This means that in terms of both econometric analysis of demand and policy design, it may be easier to know when consumption norms are influencing demand ${ }^{26}$. The implications for policies, such as the standard Pigovian taxation of goods which cause

\footnotetext{
${ }^{25}$ We are grateful to a discussant Ngo van Long for raising this point.

${ }^{26}$ Using econometric analysis of demand to detect the existence and effect of consumption norms is an indirect approach and there may be other explanations for perceived patterns of demand than the existence of a norm. There can be more direct ways of empirically investigating the existence of norms, such as the analysis by Efferson et al (2015) of female genital mutilation (FGM), which showed that FGM did not appear to be driven by a norm in specific communities.
} 
environmental damage, is that such policies may be ineffective, if the original, pre-policy, norm remains an equilibrium norm, or even counter-productive in terms of lowering welfare if the consumption norm is no longer an equilibrium norm and the loss of benefits from conformity outweigh the gains in reducing pollution damage costs.

In terms of which commodities might be associated with consumption norms, although we emphasise the difference between consumption norms and conspicuous consumption, it has to be the case that it must be possible for others to observe whether or not one is conforming to a norm, so the actual act of consumption must be conspicuous even if the motivation for adhering to a consumption norm is different from that driving conspicuous consumption.

Our model is clearly extremely simple in at least four aspects.

- Our model of consumer demand uses a simple quadratic utility function (and hence linear demand) for the norm good and linear utility for all other consumption. In earlier work (summarised in Dasgupta, Southerton, Ulph and Ulph (2016)) we used more general utility functions, and showed that the implications for individual behaviour (as summarised in Section 2 of this paper) carry over with more general utility functions.

- Our model of the costs and benefits of conforming to a norm also use particular functional forms. However, in terms of the costs of adhering to a norm, we have argued that our assumption that costs depend on the absolute value of the difference in consumption has the important implication that individuals with different levels of income will adhere exactly to the norm, which we have argued provides useful evidence that people are adhering to a consumption norm.

- Our model assumes a uniform density function of Marshallian demands, which is also clearly a special case. In our earlier work (summarised in Dasgupta, Southerton, Ulph and Ulph (2016)) we went to the other extreme of assuming that either all consumers were identical or existed in 2 or 3 groups of identical individuals. However, as we argued above, a benefit of the uniform density function is that it does not provide a fairly obvious candidate for a norm, namely the mode, so it is a more open question whether an equilibrium norm exists.

- As we noted particularly in the discussion of Results 6 and 7, our analysis does not explain how an equilibrium norm emerges, which, as we said in the introduction, is often studied using evolutionary game theory. An interesting question is whether our 
notion of an equilibrium consumption norm corresponds to the outcome of an evolutionary game.

However, while we believe there are arguments for using our particular assumptions that go beyond just the benefit of simplifying the analysis, we think it will be important for future work to explore the implications of more general assumptions about these four key features of our model. 
Figure 1: Loss of utility from adhering to a norm $\left(x^{*}, n^{*}\right)$

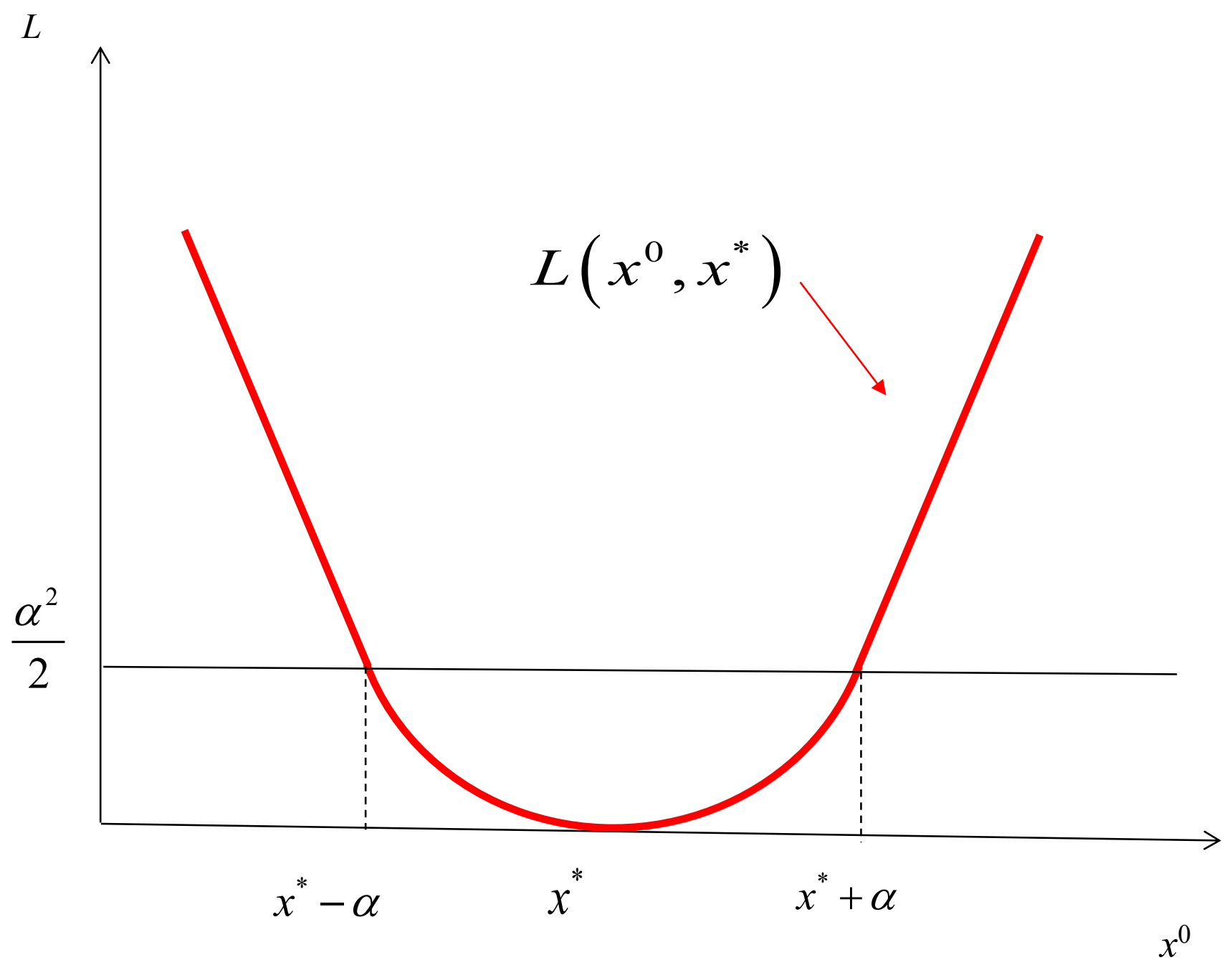


Figure 2(A) Consumption Decisions for those Adhering to Norm: Case A

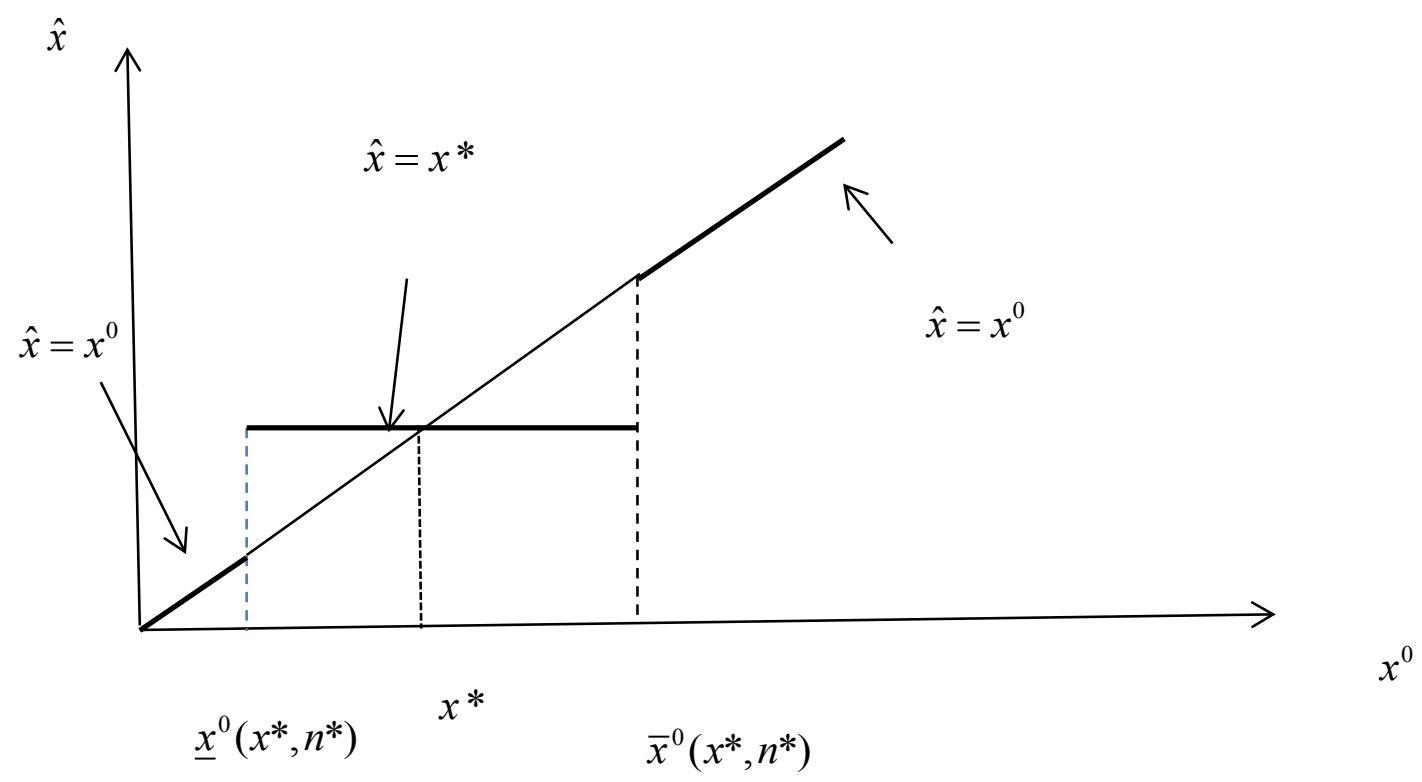


Figure 3(A) Loss of Utility from Adhering to Norm: Case A

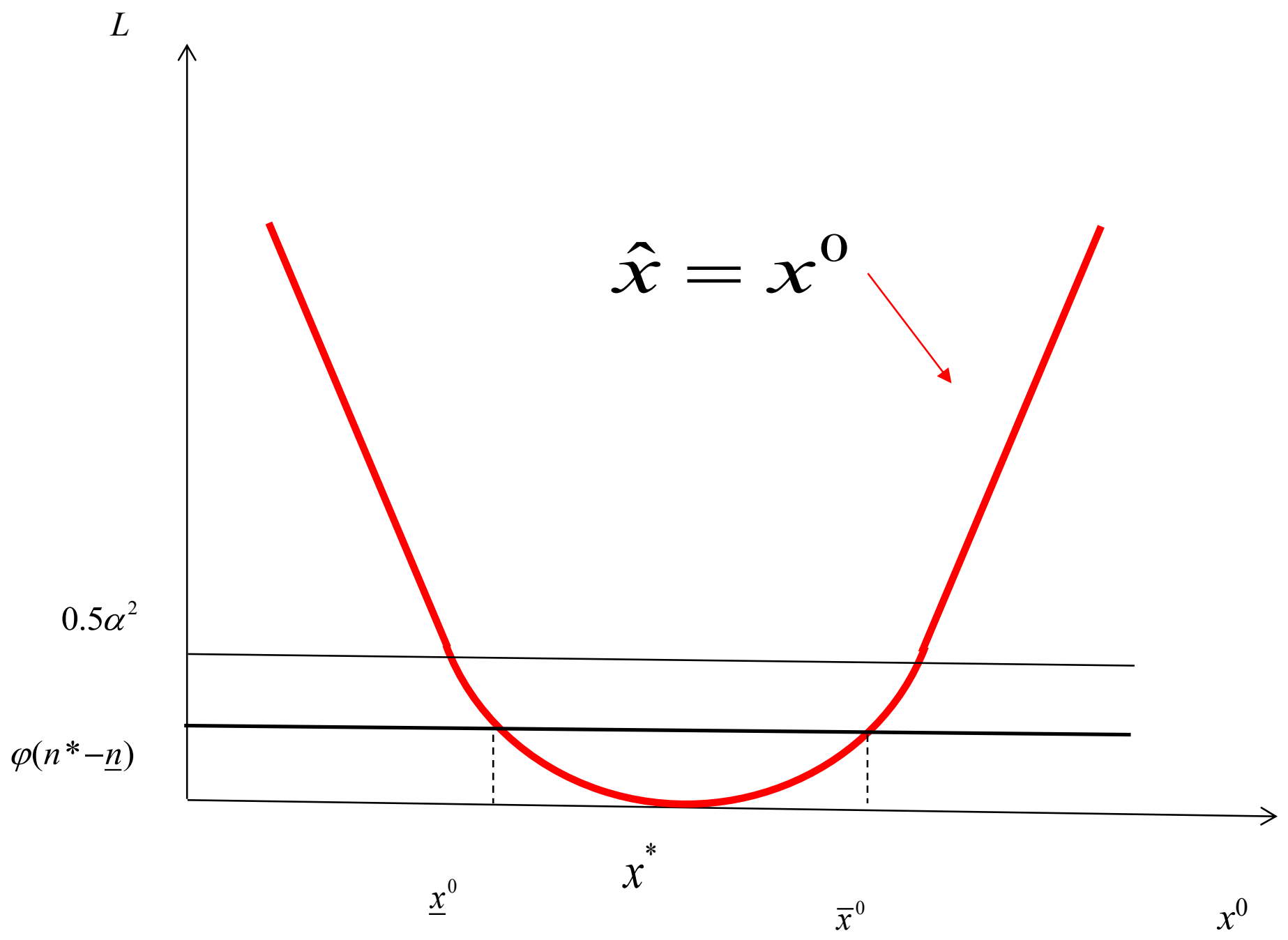


Figure 2(B) Consumption Decisions of those Adhering to Norm: Case B

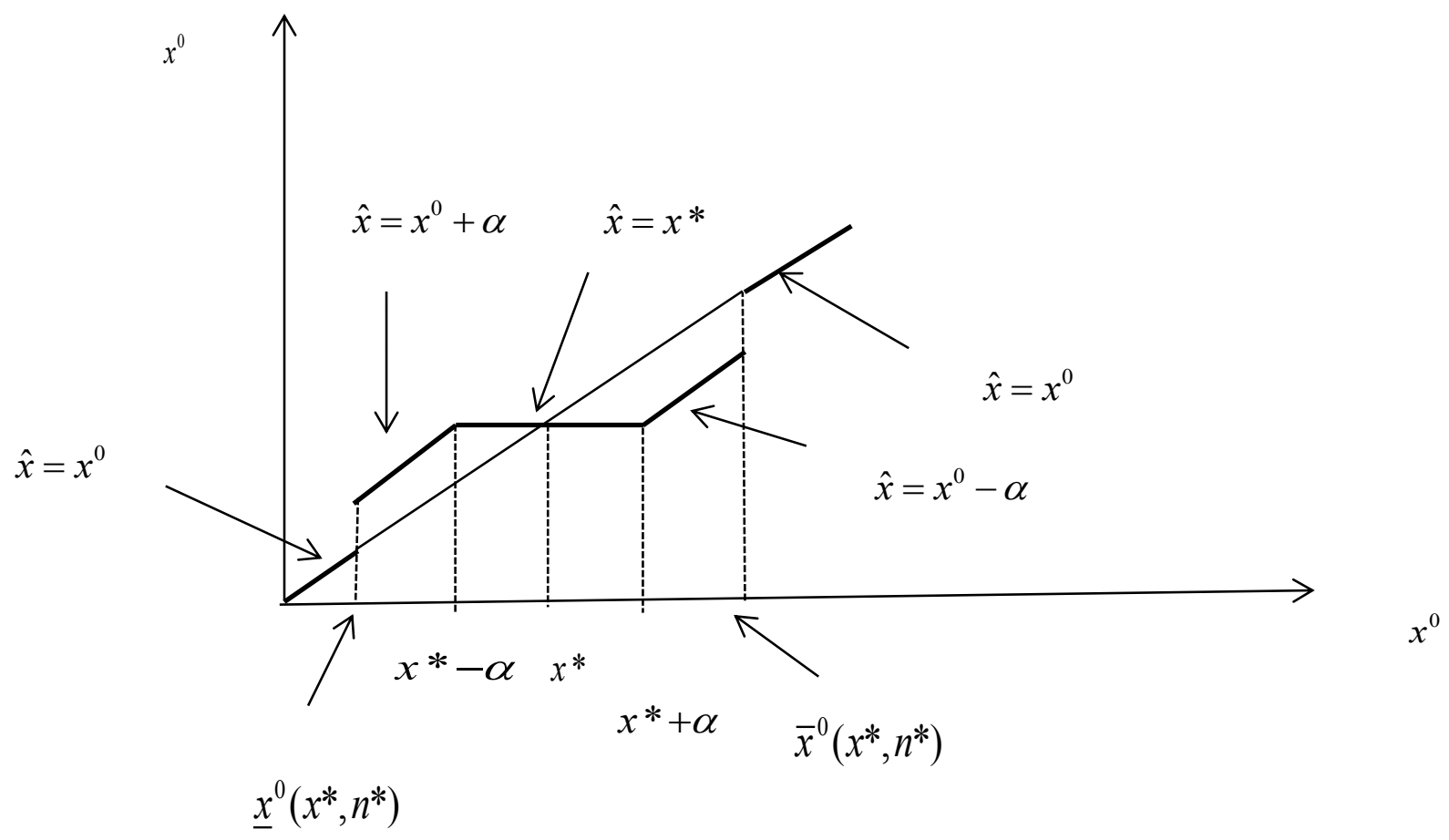


Figure 3(B) Loss of Utility from Adhering to Norm: Case B

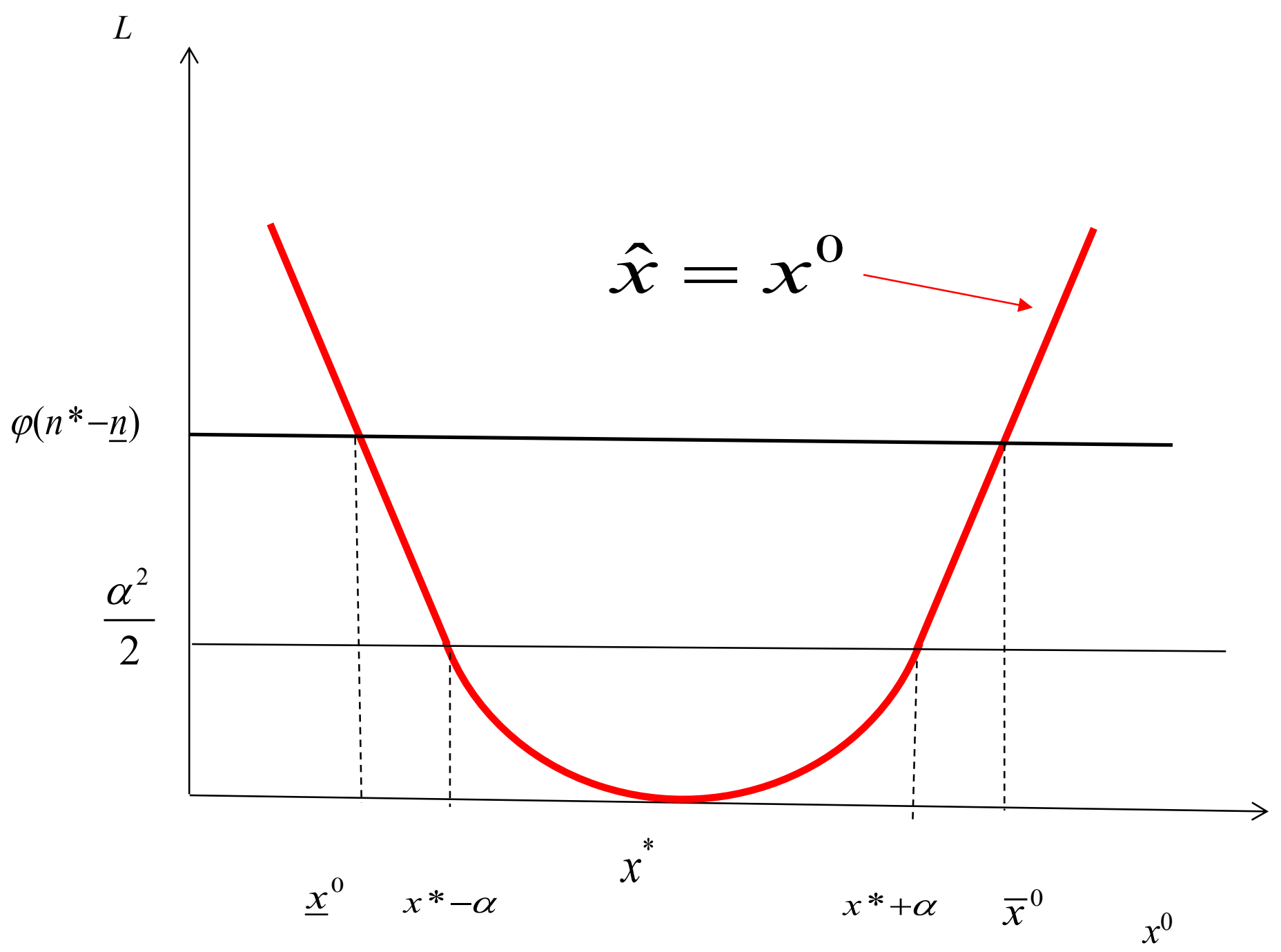


Figure 4: Equilibrium Norms: Case A $-\varphi(1-\underline{n}) \leq 0.5 \alpha^{2}$

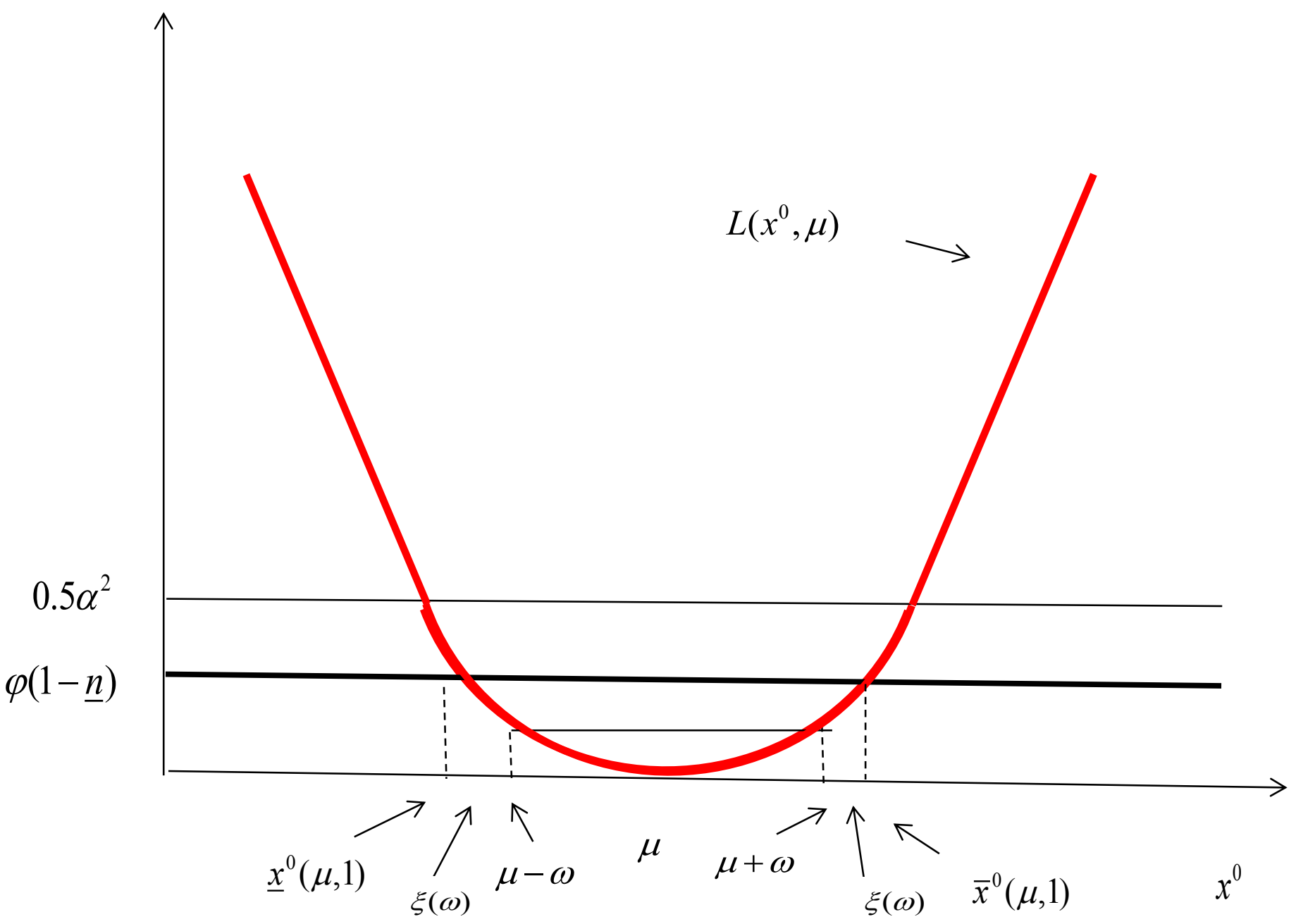


Figure 5: Equilibrium Norms: Case B(i): $\varphi(1-\underline{n})>0.5 \alpha^{2} \geq 0.5 \omega^{2}$

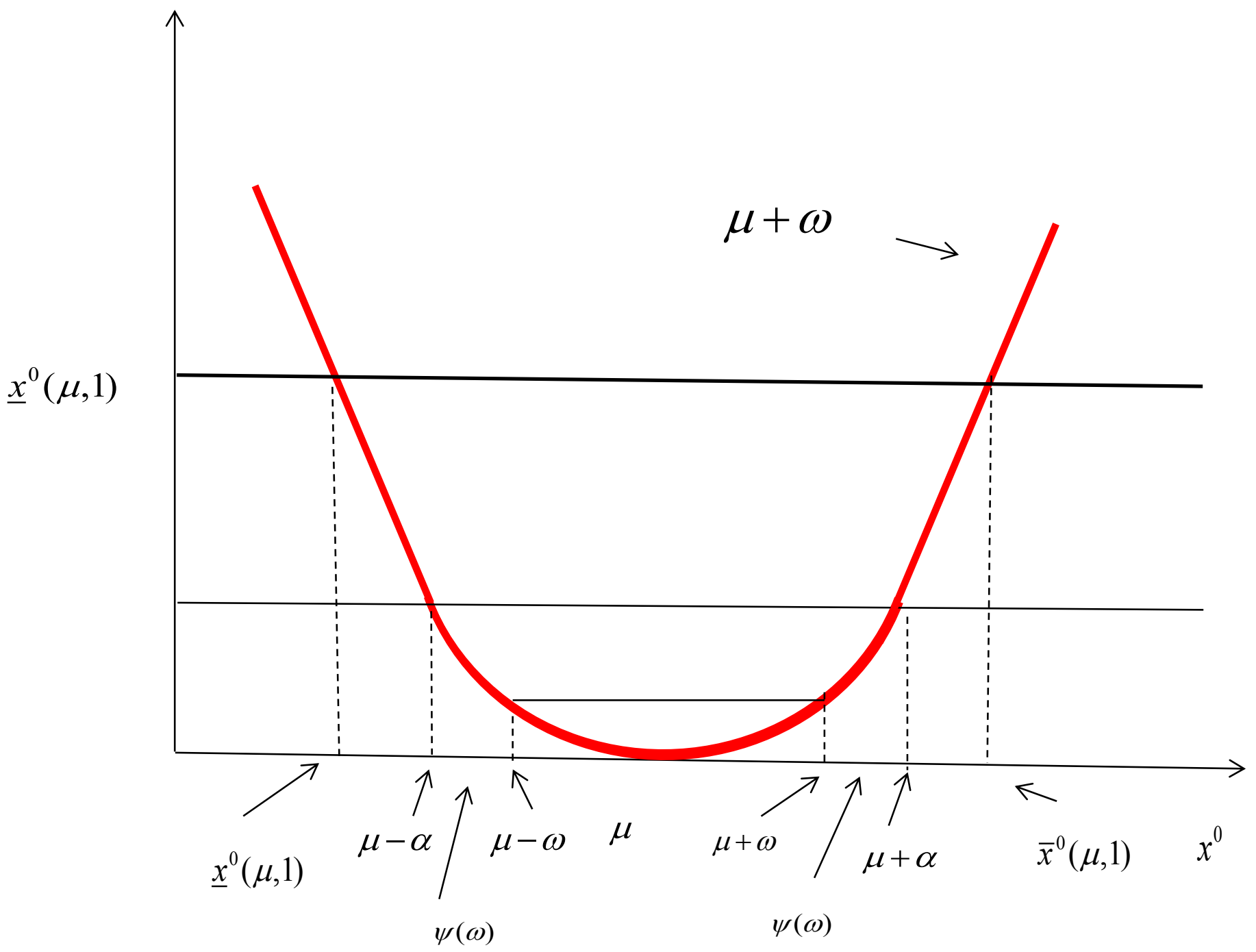


Figure 6: Equilibrium Norms: Case B(ii): $\varphi(1-\underline{n}) \geq-0.5 \alpha^{2}+\alpha \omega>0.5 \alpha^{2}$

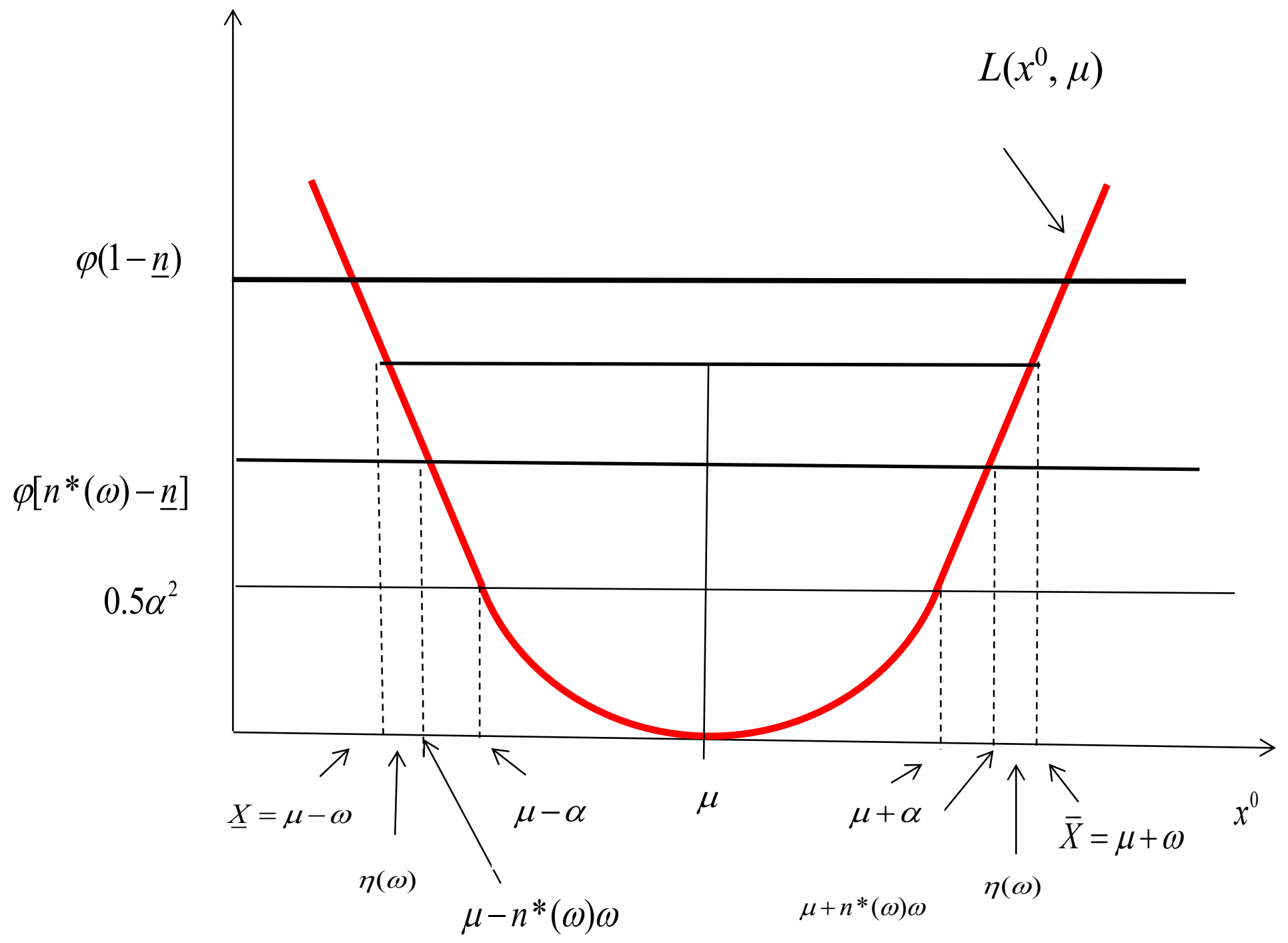


Figure 7 Case B(ii): $\varphi(1-\underline{n}) \geq-0.5 \alpha^{2}+\alpha \omega>0.5 \alpha^{2} \quad$ First Equilibrium Norm

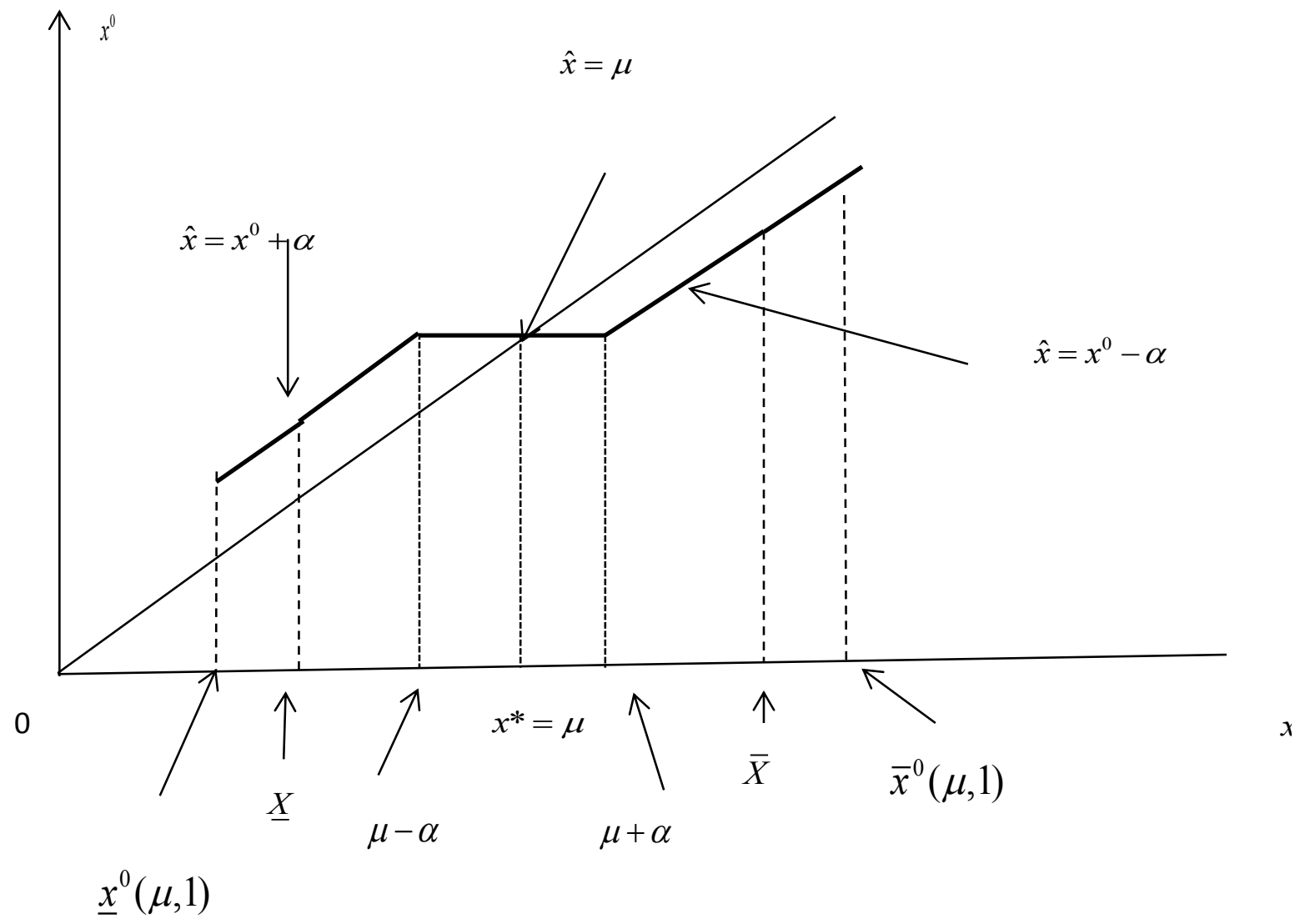


Figure 8 Case B(ii): $\varphi(1-\underline{n}) \geq-0.5 \alpha^{2}+\alpha \omega>0.5 \alpha^{2}$; Second Equilibrium Norm

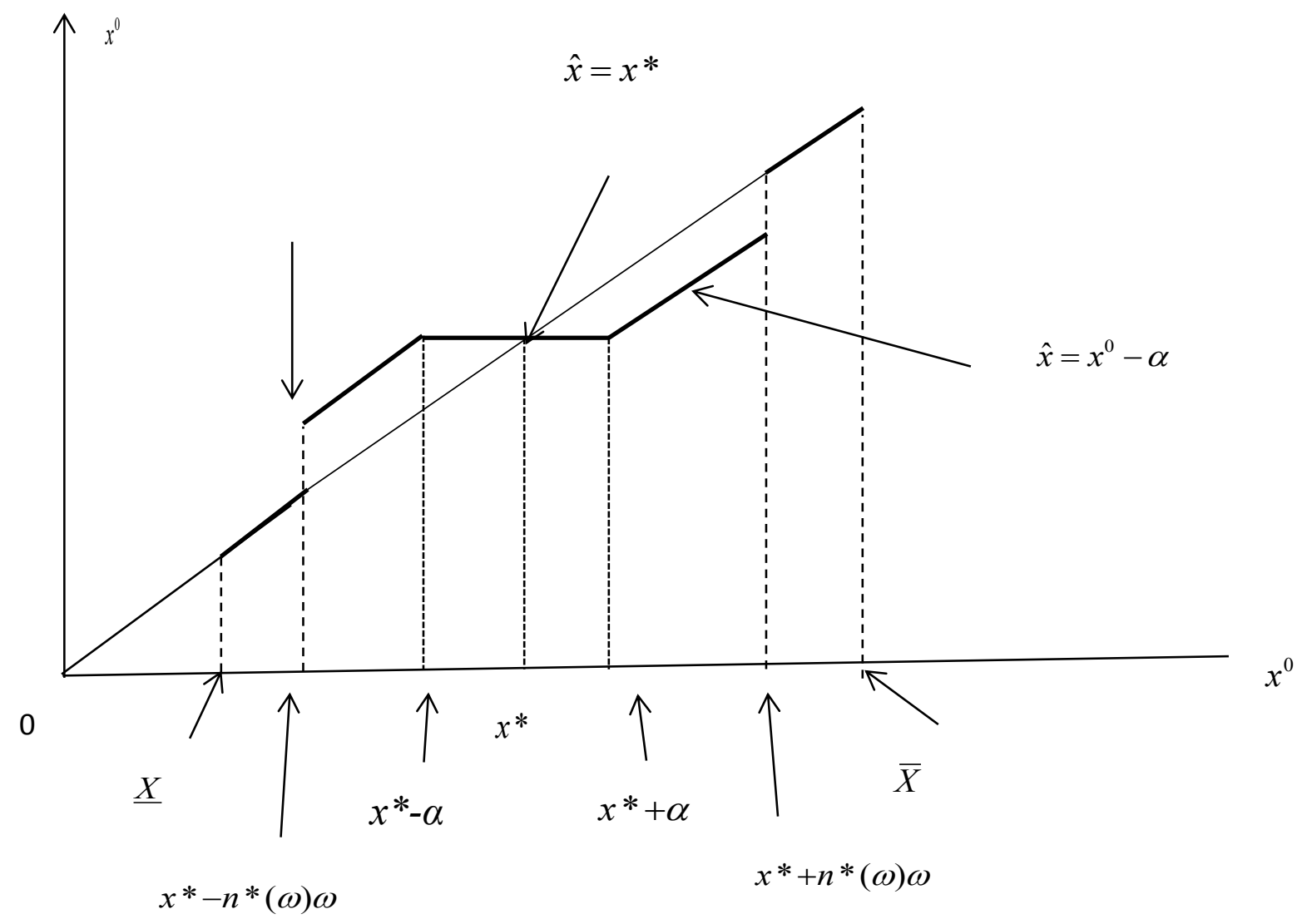




\section{References}

Abbott, A., S. Nandeibam and L. O'Shea (2013) "Recycling: Social Norms and Warm Glow Re-Visited", Ecological Economics, 90: 10-18.

Akerlof, G. and R. Kranton (2000) "Economics and Identity", Quarterly Journal of Economics, 115(3): 715-753.

Allcott, H. (2011) "Social Norms and Energy Conservation" Journal of Public Economic 95: 1082-1095.

Arrow, K. and P. Dasgupta (2009) "Conspicuous Consumption, Inconspicuous Leisure", The Economic Journal 119: F497-F516.

Axelrod, R. (1986) “An Evolutionary Approach to Norms” The American Political Science Review, 80(4):1095-1111.

Azar, O.H. (2004) "What Sustains Social Norms and How They Evolve? The Case of Tipping" Journal of Economic Behaviour and Organisation, 54(1), 49-64

Bauman, Z. (1990) Thinking Sociologically, Blackwell, Oxford.

Bennett, T., M. Savage, E. Silva, A. Warde, M. Gayo-Cal, and D. Wright (2009) Culture, Class, Distinction, Routledge: London.

Bernheim, D. (1994) “A Theory of Conformity”, Journal of Political Economy, 102(5): 841877.

Blanchflower, D. and A. Oswald (2004) "Well-being Over Time in Britain and the USA" Journal of Public Economics, 88(7), 1359-1368.

Bourdieu, P. (1984) Distinction: A Social Critique of the Judgement of Taste, Routledge Kegan Paul, London

Brekke,K., G. Kipperberg and K. Nyborg (2010) "Social Interaction in Responsibility Ascription: The Case of Household Recycling", Land Economics, 86(4), 766-784

Bucholz, W., J. Falkinger and D. Rubbelke (2012) "Non-Governmental Public Norm Enforcement in Large Societies as a Two-Stage Game of Voluntary Public Good provision" ANU Working Paper in Economics and Econometrics, 566.

Chaudhuri, A. (2011) "Sustaining Cooperation in Laboratory Public Good Game Experiments: a Selected Survey of the Literature" Experimental Economics, 14, 47-83

Dasgupta, P. (2000) "Economic Progress and the Idea of Social Capital", in P. Dasgupta and I. Serageldin (eds.) Social Capital: A Multi-Faceted Perspective, The World Bank, Washington DC, 325-424. 
Dasgupta, P., D. Southerton, A. Ulph and D. Ulph (2016) "Consumer Behaviour with Environmental and Social Externalities: Implications for Analysis and Policy", Environmental and Resource Economics, 65(1) 191-226

Easterlin, R. (1974) "Does Economic Growth Improve the Human Lot? Some Empirical , Essays in Honor of Moses Abramowitz, Academic Press, New York.

Easterlin,R. (2001) "Income and Happiness: Toward a Unified Theory", The Economic Journal, 111: 465-484

Efferson, C., S. Vogt, A. Elhadi, H. Ahmed and E. Fehr (2015) "Female Genital Cutting Without a Social Norm", Science, 349(6255), 1446-1447

Fehr, E and K. Schmidt (2006) "The Economics of Fairness, Reciprocity and Altruism Evidence and Economic Applications", Chapter 8 in S-C Kolm and J. M. Ythier, (eds) Handbook of Giving, Altruism and Reciprocity, Vol 1., Elsevier

Festinger, L. (1954) “A Theory of Social Comparison Processes”, Human Relations, 7, 117140 .

Frey, B. and S. Meier (2004) "Social Comparisons and Pro-Social Behaviour: Testing Conditional Cooperation in a Field Experiment" American Economic Review , 94(5): 17171722.

Gerber, A. and T. Rogers (2009) "Descriptive Social Norms and Motivation to Vote: Everybody's Voting and So Should You”, Journal of Politics, 71: 1-14.

Granovetter, M. (1978) "Threshold Models of Collective Behaviour", American Journal of Sociology, 83(6) 1420 - 1443.

Granovetter, M. and R. Soong (1986) "Threshold Models of Interpersonal Effects in Consumer Demand” Journal of Economic Behaviour and Organization, 7, 83-99.

Hargreaves-Heap, S. (2013) "Social Influences on Behaviour" in J. Mehta (ed.) Behavioural Economics in Competition and Consumer Policy, Report from ESRC Centre for Competition Policy, University of East Anglia.

Hargreaves-Heap, S. and D. Zizzo (2009) "The Value of Groups", American Economic Review, 99: 295-323.

Hegselmann, R. and U. Krause (2002) "Opinion Dynamics and Bounded Confidence Models, Analysis and Simulation", Journal of Artificial Societies and Social Simulation, 5(3), 1-33.

Layard, R. (2006) "Happiness and Public Policy: a Challenge to the Profession", The Economic Journal, 116: C24-C33.

Ledyard, J. (1995) "Public Goods: A Survey of Experimental Research" in J. Kregel and A. Roth (eds) Handbook of Experimental Economics, Princeton University Press, 111-252. 
Manski, C. (2000), "Economic Analysis of Social Interactions", Journal of Economic Perspectives, 14(3), 115-136.

Nyborg, K. and M. Rege (2003) “On Social Norms: the Evolution of Considerate Smoking Behaviour” Journal of Economic Behaviour and Organisation, 52, 323-340.

Perino, G., L.Panzone and T. Swanson (2014) "Motivating Crowding in Real Consumption Decisions: Who Is Messing with My Groceries”, Economic Inquiry, 52 (2), 592-607.

Putnam, R. (2000) Bowling Alone: The Collapse and Revival of American Community, Simon and Schuster, New York.

Qin,B. and T. Janus (2018) "Social Norms and the Effect of Unilateral Actions on Climate Change", mimeo, Jinhe Center for Economic Research, Xian Jiaotong University.

Schelling, T. (1971) “Dynamic Models of Segregation”, Journal of Mathematical Sociology, $1,143-186$.

Slack, S. and D. Ulph (2016), "Subjective Well-Being, Peer Comparison and Optimal Income Taxation", EconDiscPaper 1614, St. Andrews Research Repository.

Southerton, D. (2002), 'Boundaries of 'Us' and 'Them': class, mobility and identification in a new town', Sociology, 36(1): 171-193.

Ulph, D. (2014), "Keeping up with the Jones: Who loses out?" Economic Letters 125(3), 400-403.

Veblen, T. (1924) The Theory of the Leisure Class: An Economic Study of Institutions, George, Allen and Unwin, London

Young, H. P. (2015) “The Evolution of Social Norms", Annual Review of Economics, 7: 359-387. 


\section{Appendix: Proofs of Results}

$\underline{\text { Result 1: }}$ Case A $\varphi(1-\underline{n}) \leq 0.5 \alpha^{2}$

Define: $\xi(\omega) \equiv \sqrt{2 \varphi(1-\underline{n})}-\omega$

(i) If $\omega<2 \omega \leq \sqrt{2 \varphi(1-\underline{n})} \leq \alpha$ then for any $x^{*} \in[\underline{X}, \bar{X}], \quad\left(x^{*}, 1\right)$ is an equilibrium norm to which everyone adheres exactly.

(ii) If $\omega<\sqrt{2 \varphi(1-\underline{n})} \leq \min (\alpha, 2 \omega)$ then for any $x^{*} \in[\mu-\xi, \mu+\xi] \quad\left(x^{*}, 1\right)$ is an equilibrium norm to which everyone adheres exactly; as $\omega$ increases $\xi$ falls.

(iii) If $\omega=\sqrt{2 \varphi(1-\underline{n})} \leq \min (\alpha, 2 \omega)$ then $(\mu, 1)$ is the unique equilibrium norm to which everyone adheres exactly.

\section{Proof:}

(a) Suppose first that $\omega \leq \sqrt{2 \varphi(1-\underline{n})}$; then

$$
\underline{x}^{0}(\mu, 1)=\mu-\sqrt{2 \varphi(1-\underline{n})} \leq \mu-\omega=\underline{X} ; \quad \bar{x}^{0}(\mu, 1)=\mu+\sqrt{2 \varphi(1-\underline{n})} \geq \mu+\omega=\bar{X}
$$

so $\forall x^{0} \in[\underline{X}, \bar{X}]$ benefit of adhering to norm $(\mu, 1), \varphi(1-\underline{n})$, is at least as great as cost to marginal individual, $0.5 \omega^{2}$. So everyone will adhere to this norm; i.e. $n^{*}=1$ and $\hat{x}\left(x^{0}, \mu\right)=\mu \quad \forall x^{0}: \underline{X} \leq x^{0} \leq \bar{X}$, which satisfies the condition $\frac{1}{2 \omega} \int_{\underline{X}}^{\bar{X}} \hat{x}\left(x^{0}, \mu\right) d x^{0}=\mu$.

So $(\mu, 1)$ is an equilibrium norm.

(b) Suppose again that $\omega \leq \sqrt{2 \varphi(1-\underline{n})}$; for what other values of $x^{*}$ would $\left(x^{*}, 1\right)$ be an equilibrium norm? We require that the equivalent condition to (A1) is satisfied; i.e.

$$
\begin{aligned}
& \underline{x}^{0}\left(x^{*}, 1\right)=x^{*}-\sqrt{2 \varphi(1-\underline{n})} \leq \mu-\omega=\underline{X} ; \quad \bar{x}^{0}\left(x^{*}, 1\right)=x^{*}+\sqrt{2 \varphi(1-\underline{n})} \geq \mu+\omega=\bar{X} \\
& \text { i.e. } \quad \mu-\sqrt{2 \varphi(1-\underline{n})}+\omega \leq x^{*} \leq \mu+\sqrt{2 \varphi(1-\underline{n})}-\omega
\end{aligned}
$$

So:

(i) If $\xi(\omega) \geq \omega \Leftrightarrow \sqrt{2 \varphi(1-\underline{n})} \geq 2 \omega$ then for any $x^{*} \in[\underline{X}, \bar{X}]\left(x^{*}, 1\right)$ is an equilibrium norm to which everyone adheres.

(ii) If $\xi(\omega)<\omega$ then for any $x^{*} \in[\mu-\xi(\omega), \mu+\xi(\omega)]\left(x^{*}, 1\right)$ is an equilibrium norm to which everyone adheres.

(iii) If $\xi(\omega)=0$ then $(\mu, 1)$ is the unique equilibrium norm to which everyone adheres. 
(c) Suppose $\sqrt{2 \varphi(1-\underline{n})}<\omega \leq \alpha$ then obviously (A1) is no longer satisfied, so $(\mu, 1)$ cannot be an equilibrium norm .

(d) Finally we show that for all values of $\omega \leq \alpha$ there cannot be an equilibrium norm $\left(x^{*}, n^{*}\right)$ where $\underline{n}<n^{*}<1$. We show that this is true for $x^{*}=\mu$, and by extension this is true for any $x^{*} \in[\underline{X}, \bar{X}]$

If $\left(\mu, n^{*}\right)$ was an equilibrium norm then the benefit of adhering to this norm must just equal the cost to the marginal individual of adhering to this norm; i.e.

$$
2 \varphi\left(n^{*}-\underline{n}\right)=\left(n^{*} \omega\right)^{2} \Leftrightarrow n^{*}=\frac{\varphi \pm \sqrt{\varphi^{2}-2 \varphi \omega^{2} \underline{n}}}{\omega^{2}}
$$

We need to check whether $1>n^{*}>\underline{n}$. Solving (A4) for $n^{*}$ we have:

$$
n^{*}<1 \Leftrightarrow \sqrt{\varphi^{2}-2 \varphi \omega^{2} \underline{n}}<\omega^{2}-\varphi \Leftrightarrow \omega^{2}>2 \varphi(1-\underline{n})
$$

So this is not the case when $\omega \leq \sqrt{2 \varphi(1-\underline{n})} \leq \alpha$. So $\left(\mu, n^{*}\right)$ cannot be an equilibrium norm with $n^{*}<1$ when $\omega \leq \sqrt{2 \varphi(1-\underline{n})} \leq \alpha$.

So suppose (A5) holds, i.e. $\sqrt{2 \varphi(1-\underline{n})}<\omega \leq \alpha$ Then we need to check whether $n^{*}>\underline{n}$. We have:

$$
n^{*}>\underline{n} \Leftrightarrow \sqrt{\varphi^{2}-2 \varphi \omega^{2} \underline{n}}>\omega^{2} \underline{n}-\varphi \Leftrightarrow \varphi^{2}-2 \varphi \omega^{2} \underline{n}>\omega^{4} \underline{n}^{2}-2 \varphi \omega^{2} \underline{n}+\varphi^{2} \Leftrightarrow \omega^{4} \underline{n}^{2}<0
$$

which is not the case.

Putting together (c) and (d) we have shown that the unique equilibrium norms established in cases (i) - (iii) are indeed the only unique equilibrium norms and that:

(iv) If $\sqrt{2 \varphi(1-\underline{n})}<\omega \leq \alpha$ then there is no equilibrium norm.

QED

Result 2: Case B (i) $\varphi(1-\underline{n})>0.5 \alpha^{2} \geq 0.5 \omega^{2}$.

Define $\psi(\omega) \equiv \alpha-\omega$

(i) If $\omega \leq 0.5 \alpha$ then for any $x^{*} \in[\underline{X}, \bar{X}],\left(x^{*}, 1\right)$ is an equilibrium norm to which everyone adheres exactly.

(ii) If $0.5 \alpha<\omega<\alpha$, then for any $x^{*} \in[\mu-\psi(\omega), \mu+\psi(\omega)],\left(x^{*}, 1\right)$ is an equilibrium norm to which everyone adheres exactly; as $\omega$ increases $\psi$ falls. 
(iii) If $\omega=\alpha$ then $(\mu, 1)$ is the unique equilibrium norm to which everyone adheres exactly.

Proof: The proof is the same as for Result 1.

Result 3: Case B (ii) $\varphi(1-\underline{n}) \geq-0.5 \alpha^{2}+\alpha \omega>0.5 \alpha^{2}$

Define: (a) $\bar{\omega}=\frac{\varphi(1-\underline{n})}{\alpha}+0.5 \alpha$; (b) $n^{*}(\omega)=\frac{\varphi \underline{n}-0.5 \alpha^{2}}{\varphi-\alpha \omega}$; (c) $\eta(\omega)=\omega\left(1-n^{*}(\omega)\right)$

(i) For $\alpha<\omega<\bar{\omega}$ there are two possible equilibrium norms: $(\mu, 1)$ and $\left(x^{*}, n^{*}(\omega)\right)$ where $x^{*} \in[\mu-\eta(\omega), \mu+\eta(\omega)]$.

(ii) For $\omega=\bar{\omega}$, the only equilibrium norm is $(\mu, 1)$.

(iii) For $\omega>\bar{\omega}$ there is no equilibrium norm.

In cases (i) and (ii) individuals with Marshallian demands $x^{0} \in\left[x^{*}-\alpha, x^{*}+\alpha\right]$ adhere exactly to the norm $x^{*}$; individuals with Marshallian demands $x^{0} \in\left[x^{*}-n^{*}(\omega) \omega, x^{*}-\alpha\right]$ adhere by setting $\hat{x}=x^{0}+\alpha$; individuals with Marshallian demands $x^{0} \in\left[x^{*}+\alpha, x^{*}+n^{*}(\omega) \omega\right]$ adhere by setting $\hat{x}=x^{0}-\alpha$.

\section{Proof:}

(a) We first show that for $\alpha<\omega \leq \bar{\omega}(\mu, 1)$ is an equilibrium norm.

From the assumption on parameter values for Result 3

$$
\underline{x}^{0}(\mu, 1)=\mu-0.5 \alpha-\frac{\varphi}{\alpha}(1-\underline{n})<\underline{X} \Leftrightarrow \varphi(1-\underline{n})>\alpha(\omega-0.5 \alpha)
$$

Similarly $\bar{x}^{0}(\mu, 1)>\bar{X}$. So $\forall x^{0}, \quad \mu-\omega=\underline{X} \leq x^{0} \leq \bar{X}=\mu+\omega$ so every consumer the gain from abiding by the norm $(\mu, 1), \varphi(1-\underline{n})$, is at least as great as the cost of abiding by that norm, $\alpha(\omega-0.5 \alpha)$; so everyone abides by the norm $(\mu, 1)$; hence $n^{*}=1$. Average consumption is given by: 


$$
\begin{aligned}
& \frac{1}{2 \omega}\left\{\int_{\mu-\omega}^{\mu-\alpha}\left(x^{0}+\alpha\right) d x^{0}+\int_{\mu-\alpha}^{\mu+\alpha} \mu d x^{0}+\int_{\mu+\alpha}^{\mu+\omega}\left(x^{0}-\alpha\right) d x^{0}\right\} \\
& =\frac{1}{2 \omega}\left\{0.5(\mu-\alpha)^{2}+\alpha(\mu-\alpha)-0.5(\mu-\omega)^{2}-\alpha(\mu-\omega)+2 \alpha \mu\right. \\
& \left.+0.5(\mu+\omega)^{2}-\alpha(\mu+\omega)-0.5(\mu+\alpha)^{2}+\alpha(\mu+\alpha)\right\} \\
& =\frac{2 \mu \omega}{2 \omega}=\mu
\end{aligned}
$$

So average consumption equals the norm. So $(\mu, 1)$ is an equilibrium norm.

(b) We now show that $\left(x^{*}, 1\right)$ cannot be an equilibrium norm with $x^{*} \neq \mu$.

Suppose that $x^{*}$ shifts slightly away from $\mu$ (the same argument applies for more extreme changes in norm, as we shall argue). Then if everyone adheres to the norm ( $\left.x^{*} 1\right)$, average consumption is now

$$
\begin{aligned}
& \frac{1}{2 \omega}\left\{\int_{\mu-\omega}^{x^{*}-\alpha}\left(x^{0}+\alpha\right) d x^{0}+\int_{x^{*}-\alpha}^{x^{*}+\alpha} x^{*} d x^{0}+\int_{x^{*}+\alpha}^{\mu+\omega}\left(x^{0}-\alpha\right) d x^{0}\right\} \\
& =\frac{1}{2 \omega}\left\{0.5\left(x^{*}-\alpha\right)^{2}+\alpha\left(x^{*}-\alpha\right)-0.5(\mu-\omega)^{2}-\alpha(\mu-\omega)+2 \alpha x^{*}\right. \\
& \left.+0.5(\mu+\omega)^{2}-\alpha(\mu+\omega)-0.5\left(x^{*}+\alpha\right)^{2}+\alpha\left(x^{*}+\alpha\right)\right\} \\
& =\frac{\mu \omega+\alpha\left(x^{*}-\mu\right)}{\omega} \neq x^{*}
\end{aligned}
$$

So $\left(x^{*}, 1\right)$ cannot be an equilibrium norm. Hence $(\mu, 1)$ is not a stable equilibrium norm. This argument is even stronger if a possible norm is sufficiently different from $\mu$ that one of groups who do not adhere exactly to the norm no longer lies in the range $[\underline{X}, \bar{X}]$. So $(\mu, 1)$ is a possible equilibrium norm. But any change in parameter values which led to a different value for $\mu$, call it $\hat{\mu}$, would mean the original norm $(\mu, 1)$ is no longer an equilibrium norm.

(c) We now assume $\alpha<\omega \leq \bar{\omega}$ and ask whether $\left(\mu, n^{*}(\omega)\right)$ could be a stable norm for some value of $n^{*}(\omega)$.

For that to be an equilibrium norm, the benefit to the marginal person adhering to the norm, $\varphi(n *(\omega)-\underline{n})$ must equal the cost $-0.5 \alpha^{2}+\alpha n^{*}(\omega) \omega$, i.e.

$$
n *(\omega)=\frac{\varphi \underline{n}-0.5 \alpha^{2}}{\varphi-\alpha \omega}
$$

We first show that $n *(\omega) \leq 1$. So

$$
n^{*}(\omega) \leq 1 \Leftrightarrow \varphi(1-\underline{n}) \geq 0.5 \alpha(2 \omega-\alpha)
$$

which is true by the assumption on parameter values for Result 3. We now show that $n^{*}>\underline{n}$. 
From (A9) $\varphi>\frac{0.5 \alpha(2 \omega-\alpha)}{1-\underline{n}}$; furthermore $\frac{0.5 \alpha(2 \omega-\alpha)}{1-\underline{n}}>\alpha \omega \Leftrightarrow \underline{n}>\frac{\alpha}{2 \omega}$ which is true since $\underline{n} \geq 0.5$. So $\varphi>\alpha \lambda$. Then:

$$
n^{*}(\omega)>\underline{n} \Leftrightarrow \varphi \underline{n}-0.5 \alpha^{2}>\varphi \underline{n}-\alpha \omega \underline{n} \Leftrightarrow \underline{n}>\frac{\alpha}{2 \omega} \text { which is true since } \underline{n}>0.5>\frac{\alpha}{2 \omega}
$$

So we have proved $\underline{n}<n^{*}(\omega) \leq 1$.

To establish that $\left(\mu, n^{*}\right)$ is a norm we need to show that $\mu$ is the average of $\hat{x}\left(x^{0}, \mu\right)$ over the range $\left[\mu-n^{*}(\omega) \omega, \mu+n^{*}(\omega) \omega\right]$. For ease of notation define $\lambda \equiv n^{*}(\omega) \omega$. Then the average value of $\hat{x}\left(x^{0}, \mu\right)$ over the range $[\mu-\lambda, \mu+\lambda]$ is:

$$
\begin{aligned}
& \frac{1}{2 \lambda}\left\{\int_{\mu-\lambda}^{\mu-\alpha}\left(x^{0}+\alpha\right) d x^{0}+\int_{\mu-\alpha}^{\mu+\alpha} \mu d x^{0}+\int_{\mu+\alpha}^{\mu+\lambda}\left(x^{0}-\alpha\right) d x^{0}\right\}= \\
& \frac{1}{2 \lambda}\left\{0.5(\mu-\alpha)^{2}+\alpha(\mu-\alpha)-0.5(\mu-\lambda)^{2}-\alpha(\mu-\lambda)+2 \alpha \mu\right. \\
& \left.+0.5(\mu+\lambda)^{2}-\alpha(\mu+\lambda)-0.5(\mu+\alpha)^{2}+\alpha(\mu+\alpha)\right\}=\frac{1}{2 \lambda}\{2 \lambda \mu\}=\mu
\end{aligned}
$$

So $(\mu, n *(\omega))$ is an equilibrium norm.

Finally we show that for any $x^{*} \in[\mu-\eta(\omega), \mu+\eta(\omega)]$, where $\eta(\omega)=\omega\left(1-n^{*}(\omega)\right),\left(x^{*}\right.$, $n *(\omega))$ is an equilibrium norm. $\eta(\omega)$ is defined by the condition that:

$$
\left.x^{*}-n^{*}(\omega) \omega=\mu-\omega=\underline{X} \text { (equivalently } x^{*}-n^{*}(\omega) \omega=\mu-\omega=\underline{X}\right) .
$$

(d) Finally note that:

$$
n^{* \prime}(\omega)>0 ; \quad \underline{n}<n^{*}(\alpha)<1 ; \quad n^{*}(\bar{\omega})=1 ; \eta(\alpha)=\alpha\left(1-n^{*}(\alpha)\right)>0 ; \eta(\bar{\omega})=0
$$

If $\hat{\omega}>\bar{\omega}$ it would have to be the case that $n^{*}(\hat{\omega})<1$ but from (A12) $n^{*}(\hat{\omega})>1$, which is contradiction, so if $\hat{\omega}>\bar{\omega}$ there is no equilibrium norm.

QED

Result 4: Suppose (a) $0.5 \alpha+\varphi(1-\underline{n}) / \alpha>\omega>\max [1.5 \alpha, \alpha+\varphi(1-2 \underline{n}) / \alpha] \quad$ and $\varphi \geq 1.5 \alpha^{2} \Rightarrow \underline{n}>1 / 3 \geq 0.5 \alpha^{2} / \varphi$ and define: $\rho(\omega)=n *(\omega) \omega$. Then:

(i) $0.5>n *(\omega)>\underline{n}>1 / 3$

(ii) If $\mu+\omega-3 \rho(\omega)<x^{*}<\mu-\omega+3 \rho(\omega)$ then $\left(x^{*}, n^{*}(\omega)\right)$ is the unique equilibrium norm. 
(iii) If $\mu-\omega<x^{*}<\mu+\omega-3 \rho(\omega) \Rightarrow \mu+\omega-\left(x^{*}+\rho(\omega)\right)>2 \rho(\omega) \quad-\quad$ so there is sufficient room for a second norm $\left(x^{* *}, n(\omega)\right)$ with $x^{* *}>x^{*}$; similarly if $\mu+\omega>x^{*}>\mu-\omega+3 \rho(\omega) \Rightarrow\left(x^{*}-\rho(\omega)\right)-(\mu-\omega)>2 \rho(\omega)$ - so there is sufficient room for a second norm $\left(x^{* *}, n(\omega)\right)$ with $x * *<x^{*}$.

\section{Proof:}

(i) Result 3 holds iff $\varphi(1-\underline{n})>-0.5 \alpha^{2}+\alpha \omega>0.5 \alpha^{2} \Leftrightarrow \frac{\varphi(1-\underline{n})}{\alpha}>\omega>\alpha$. (A13)

$$
\begin{aligned}
& n *(\omega)=\frac{\varphi \underline{n}-0.5 \alpha^{2}}{\varphi-\alpha \omega}<0.5 \Leftrightarrow \alpha(\omega-\alpha)>\varphi(1-2 \underline{n}) \Leftrightarrow \omega>\frac{\varphi(1-2 \underline{n})}{\alpha}+\alpha>\alpha \text { (A14) } \\
& n^{*}(\omega)>\underline{n} \Leftrightarrow \underline{n}>0.5 \alpha / \omega ; \text { to ensure } \underline{n}>1 / 3 \text { we require } \omega>1.5 \alpha
\end{aligned}
$$

Combining these conditions yields conditions (a) and (b) above. Hence if (a) and (b) hold, Result 3 ensures that $\left(x^{*}, n^{*}(\omega)\right)$ can be an equilibrium norm with $0.5>n *(\omega)>\underline{n}>1 / 3$.

(ii) Note first that $0<3 \rho(\omega)-\omega<\omega \Leftrightarrow n^{*}(\omega)<2 / 3$, which clearly holds. Then if $\mu+\omega-3 \rho(\omega)<x^{*}<\mu-\omega+3 \rho(\omega), \mu+\omega-\left(x^{*}+\rho(\omega)\right)<2 \rho(\omega)$ so there is insufficient space for a second norm $\left(x^{* *}, n^{*}(\omega)\right)$ with $x^{* *}>x^{*}$. Similarly $x^{*}-\rho(\omega)-(\mu-\omega)<2 \rho(\omega)$ so there is insufficient space for a second norm $\left(x^{* *}\right.$, $\left.n^{*}(\omega)\right)$ with $x^{* *}<x^{*}$.

(iii) This follows directly from (ii). QED

Result 5: In Results 1, 2, and 3 there are ranges of possible values for an equilibrium norm $x^{*}$ (with an associated value of $n^{*}$ which is either 1 or a constant independent of $x^{*}$ ). In Results 1 and 2 the value of $x^{*}$ which minimises the expected value of the utility loss $\underset{x^{0}}{E}\left[L\left(x^{0}, x^{*}\right)\right]$ is $x^{*}=\mu$. In Result 3 there is no optimal value of $x^{*}$ - the value of $\underset{x^{0}}{E}\left[L\left(x^{0}, x^{*}\right)\right]$ is the same for all possible equilibrium values of $x^{*}$.

\section{Proof:}

In Results 1 and 2 the equilibrium norm $x *$ lies in a range of possible values, namely $[\mu-\omega, \mu+\omega],[\mu-\xi(\omega), \mu+\xi(\omega)],[\mu-\psi(\omega), \mu+\psi(\omega)]$ with $n^{*}=1$, and everyone adheres exactly to the norm $x^{*}$. So: 


$$
\begin{aligned}
&{\underset{x^{0}}{E}\left[L\left(x^{*}, x^{0}\right)\right]}=\frac{0.5}{(\bar{X}-\underline{X})} \int_{\underline{X}}^{\bar{X}}\left(x^{0}-x^{*}\right)^{2} d x_{0}=\frac{0.5}{(\bar{X}-\underline{X})}\left[\frac{x^{0^{3}}}{3}-x * x^{0^{2}}+x^{* 2} x^{0}\right]_{\underline{X}}^{\bar{X}} \\
&=\frac{\left(\bar{X}^{3}-\underline{X}^{3}\right)}{6(\bar{X}-\underline{X})}-\frac{x^{*}\left(\bar{X}^{2}-\underline{X}^{2}\right)}{2(\bar{X}-\underline{X})}+\frac{x^{* 2}}{2}
\end{aligned}
$$

Hence:

$$
\frac{\partial \underset{x^{0}}{E}\left[L\left(x^{*}, x^{0}\right)\right]}{\partial x^{*}}=-\frac{\bar{X}+\underline{X}}{2}+x^{*}=0 \Rightarrow x^{*}=\frac{\bar{X}+\underline{X}}{2}=\mu
$$

In Result 3 the equilibrium norm $x^{*}$ lies in a range of possible values $[\mu-\eta(\omega), \mu+\eta(\omega)]$ with associated $n^{*}(\omega)=\frac{\varphi \underline{n}-0.5 \alpha^{2}}{\varphi-\alpha \omega}$. Define $\varsigma \equiv \frac{\alpha}{2}+\frac{\varphi[n *(\omega)-\underline{n}]}{\alpha}>\alpha$; then for those adhering to the norm $\left(x^{*}, n^{*}\right)$ the chosen levels of demand associated welfare losses are:

$$
\begin{array}{lll}
x^{*}-\varsigma \leq x^{0}<x^{*}-\alpha & \hat{x}=x^{0}+\alpha & L\left(x^{*}, x^{0}\right)=-0.5 \alpha^{2}+\alpha\left(x^{*}-x^{0}\right) \\
x^{*}-\alpha \leq x^{0} \leq x^{*}+\alpha & \hat{x}=x^{*} & L\left(x^{*}, x^{0}\right)=0.5\left(x^{0}-x^{*}\right)^{2} \\
x^{*}+\alpha<x^{0} \leq x^{*}+\varsigma & \hat{x}=x^{0}-\alpha & L\left(x^{*}, x^{0}\right)=-0.5 \alpha^{2}+\alpha\left(x^{0}-x^{*}\right)
\end{array}
$$

Hence:

$$
\begin{aligned}
\Omega \equiv & (\bar{X}-\underline{X}) \underset{x^{0}}{E}\left[L\left(x^{*}, x^{0}\right)\right]=\int_{x^{*}-\varsigma}^{x^{*}-\alpha}\left[-0.5 \alpha^{2}+\alpha\left(x^{*}-x^{0}\right)\right] d x^{0}+\int_{x^{*}-\alpha}^{x^{*}+\alpha}\left[0.5\left(x^{0}-x^{*}\right)^{2} d x^{0}\right. \\
& \quad+\int_{x^{*}+\alpha}^{x^{*}+\varsigma}\left[-0.5 \alpha^{2}+\alpha\left(x^{0}-x^{*}\right)\right] d x^{0} \\
\Omega & =\left[-0.5 \alpha^{2}+\alpha x^{*}\right]\left[x^{0}\right]_{x^{*}-\varsigma}^{x^{*}-\alpha}-0.5 \alpha\left[x^{0^{2}}\right]_{x^{*}-\varsigma}^{x^{*}-\alpha} \\
& +0.5\left[\frac{x^{0^{3}}}{3}-x^{*} x^{0^{2}}+x^{* 2} x^{0}\right]_{x^{*}-\alpha}^{x^{*}+\alpha}+\left[-0.5 \alpha^{2}-\alpha x^{*}\right]\left[x^{0}\right]_{x^{*}+\alpha}^{x^{*}+}+0.5 \alpha\left[x^{0^{2}}\right]_{x^{*}+\alpha}^{x^{*}+\varsigma} \\
\Omega & =\left[-0.5 \alpha^{2}+\alpha x^{*}\right](\varsigma-\alpha)-0.5 \alpha\left[\left(x^{*}-\alpha\right)^{2}-\left(x^{*}-\varsigma\right)^{2}\right] \\
& +0.5\left[\frac{\left(x^{*}+\alpha\right)^{3}}{3}-x^{*}\left(x^{*}+\alpha\right)^{2}+x^{* 2}\left(x^{*}+\alpha\right)-\frac{\left(x^{*}-\alpha\right)^{3}}{3}+x *\left(x^{*}-\alpha\right)^{2}-x^{* 2}\left(x^{*}-\alpha\right)\right] \\
& +\left[-0.5 \alpha^{2}-\alpha x^{*}\right](\varsigma-\alpha)+0.5 \alpha\left[\left(x^{*}+\varsigma\right)^{2}-\left(x^{*}+\alpha\right)^{2}\right]
\end{aligned}
$$

Hence: 


$$
\begin{aligned}
\frac{\partial \Omega}{\partial x^{*}} & =\alpha(\varsigma-\alpha)-\alpha\left[\left(x^{*}-\alpha\right)-\left(x^{*}-\varsigma\right)\right]+0.5\left\{\left(x^{*}+\alpha\right)^{2}-\left(x^{*}+\alpha\right)^{2}-2 x^{*}\left(x^{*}+\alpha\right)\right. \\
& \left.+2 x^{*}\left(x^{*}+\alpha\right)+x^{* 2}-\left(x^{*}-\alpha\right)^{2}+\left(x^{*}-\alpha\right)^{2}+\left(x^{*}-\alpha\right)^{2}+2 x^{*}\left(x^{*}-\alpha\right)-2 x^{*}\left(x^{*}-\alpha\right)-x^{* 2}\right\} \\
& -\alpha(\varsigma-\alpha)+\alpha\left[\left(x^{*}+\varsigma\right)-\left(x^{*}+\alpha\right)\right] \\
& =0 .
\end{aligned}
$$

QED 